\title{
SPECTRAL DENSITY ESTIMATION FOR STATIONARY STABLE RANDOM FIELDS
}

Abstract. We consider a stationary symmetric stable bidimensional process with discrete time, having the spectral representation (1.1). We consider a general case where the spectral measure is assumed to be the sum of an absolutely continuous measure, a discrete measure of finite order and a finite number of absolutely continuous measures on several lines. We estimate the density of the absolutely continuous measure and the density on the lines.

1. Introduction. A complex random variable $X=X_{1}+i X_{2}$ is symmetric $\alpha$-stable (S. $\alpha . S$ ) if its characteristic function is of the form

$$
\exp \left\{-\int_{S_{2}}\left|t_{1} x_{1}+t_{2} x_{2}\right|^{\alpha} d \Gamma_{X_{1}, X_{2}}\left(x_{1}, x_{2}\right)\right\},
$$

where $t=t_{1}+i t_{2}$ and $\Gamma_{X_{1}, X_{2}}$ is a symmetric measure on the unit sphere $S_{2}$ of $\mathbb{R}^{2}$.

A stochastic process $\left\{X_{t}, t \in T\right\}$ is (S. $\left.\alpha . S\right)$ if all linear combinations, $a_{1} X_{t_{1}}+\ldots+a_{n} X_{t_{n}}$, are (S. $\left.\alpha . S\right)$. When $X=X_{1}+i X_{2}$ and $Y=Y_{1}+i Y_{2}$ are jointly (S. $\alpha . S$ ) and $1<\alpha \leq 2$, the covariation of $X$ with $Y$ is defined in [3] by

$$
[X, Y]_{\alpha}=\int_{S_{4}}\left(x_{1}+i x_{2}\right)\left(y_{1}+i y_{2}\right)^{\langle\alpha-1\rangle} d \Gamma_{X_{1}, X_{2}, Y_{1}, Y_{2}}\left(x_{1}, x_{2}, y_{1}, y_{2}\right),
$$

where by convention, for $b>0$ and $Z$ a complex number, $Z^{\langle b\rangle}=|Z|^{b-1} Z^{*}$, $Z^{*}$ being the complex conjugate of $Z$. The quantity $\|X\|_{\alpha}=[X, X]_{\alpha}^{1 / \alpha}$ is a norm [11] on the linear space of (S. $\alpha . S)$ random variables. If $\left(\xi_{t}\right)_{t \in \mathbb{R}}$ is a complex (S. $\alpha . S)$ process with independent increments, then the measure $\mu$

1991 Mathematics Subject Classification: 62G07, 60G35.

Key words and phrases: periodogram, Jackson kernel, double kernel method, (S. $\alpha . \mathrm{S})$ process. 
defined by $\mu(] s, t])=\left\|\xi_{t}-\xi_{s}\right\|_{\alpha}^{\alpha}$ is a Lebesgue-Stieltjes measure [3], called the spectral measure of $\xi$. When $\mu$ is absolutely continuous, its density is called the spectral density of $\xi$.

For stationary complex symmetric $\alpha$-stable (S. $\alpha . \mathrm{S})$ processes having the spectral representation

$$
X(t)=\int_{-\infty}^{\infty} e^{i t \lambda} d \xi(\lambda)
$$

where $\xi$ is a (S. $\alpha . S)$ process with independent isotropic increments, the spectral density function $\phi$ is estimated in [11].

Let us consider a bidimensional (random field), complex (S. $\alpha . S)$ process in discrete time, having the spectral representation

$$
X(n, m)=\int_{-\pi}^{\pi} \int_{-\pi}^{\pi} e^{i\left(\lambda_{1} n+\lambda_{2} m\right)} d \xi\left(\lambda_{1}, \lambda_{2}\right),
$$

where $\xi$ is a (S. $\alpha . S)$ process with independent isotropic increments; that means $\xi$ is an additive complex function defined on the Borel subsets of $[-\pi, \pi]^{2}$ such that:

- for any integer $k$ and any Borel sets $B_{1}, \ldots, B_{k},\left(\xi\left(B_{1}\right), \ldots, \xi\left(B_{k}\right)\right)$ is (S. $\alpha . \mathrm{S})$;

- for any integer $k$ and any disjoint Borel sets $B_{1}, \ldots, B_{k}, \xi\left(B_{1}\right), \ldots$ $\ldots, \xi\left(B_{k}\right)$ are complex (S. $\left.\alpha . S\right)$ independent random variables;

- for all Borel sets $B$, the distribution of the random variable $e^{i \theta} \xi(B)$ is independent of $\theta$.

For more details about the spectral representation see [8] and [1].

We define the spectral measure by

$$
[\xi(B), \xi(B)]_{\alpha}=\int_{B} d \mu\left(\lambda_{1}, \lambda_{2}\right) \quad \text { for any Borel subset } B \text { of }[-\pi, \pi]^{2},
$$

where $[X, Y]_{\alpha}$ denotes the covariation of $X$ with $Y$ [3] when $X$ and $Y$ are jointly (S. $\alpha . S)$.

To our knowledge the case we deal with is more general than those considered in [11], [6]: we suppose that the spectral measure of the process is the sum of an absolutely continuous measure, a discrete measure of finite order and a finite number of absolutely continuous measures on several lines:

$$
\begin{aligned}
d \mu\left(\lambda_{1}, \lambda_{2}\right)= & \phi\left(\lambda_{1}, \lambda_{2}\right) d \lambda_{1} d \lambda_{2}+\sum_{j=1}^{q} a_{j}^{\prime} \delta_{\left(w_{1 j}, w_{2 j}\right)} \\
& +\sum_{i=1}^{q^{\prime}} \phi_{i}\left(u_{1}\right) \delta_{u_{2}=a_{i} u_{1}+b_{i}},
\end{aligned}
$$


where $\phi$ and $\phi_{i}$ for $i=1, \ldots, q^{\prime}$ are nonnegative continuous functions, $a_{j}^{\prime} \in$ $\mathbb{R}^{+}, a_{i}, b_{i} \in \mathbb{R}$ and $w_{1 j}, w_{2 j} \in[-\pi, \pi], j=1, \ldots, q, i=1, \ldots, q^{\prime}$.

Using Jackson polynomial kernels and the double kernel method, we estimate the function $\phi\left(\lambda_{1}, \lambda_{2}\right)$ for every $\left(\lambda_{1}, \lambda_{2}\right)$ in $]-\pi, \pi\left[^{2}\right.$. Under appropriate conditions on the function $\phi$, we obtain rates of convergence. Let us also indicate that our methods allow us to estimate the positive numbers $a_{j}^{\prime}(j=1, \ldots, q)$ and the functions $\phi_{i}\left(i=1, \ldots, q^{\prime}\right)$. For brevity, we consider here only the estimation of $\phi$; for details, see [13].

2. The periodogram and its statistical properties. Given $N M$

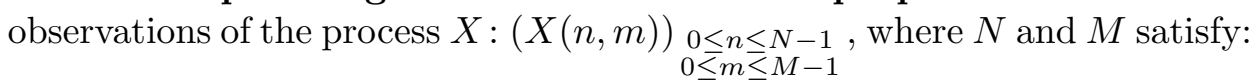

- $N-1=2 k(n-1)$ with $n \in \mathbb{N}, k \in \mathbb{N} \cup\{1 / 2\}$; if $k=1 / 2$ then $n=2 n_{1}-1, n_{1} \in \mathbb{N}$.

- $M-1=2 k(m-1)$ with $m \in \mathbb{N}, k \in \mathbb{N} \cup\{1 / 2\}$; if $k=1 / 2$ then $m=2 m_{1}-1, m_{1} \in \mathbb{N}$.

We consider the function

$$
H^{(N)}(\lambda)=\sum_{m^{\prime}=-k(n-1)}^{k(n-1)} h_{k}\left(m^{\prime} / n\right) \cos \left(\lambda m^{\prime}\right),
$$

where $h_{k}$ is defined in [7] such that

$$
H^{(N)}(\lambda)=\frac{1}{q_{k, n}}\left(\frac{\sin \frac{n \lambda}{2}}{\sin \frac{\lambda}{2}}\right)^{2 k} \quad \text { with } \quad q_{k, n}=\frac{1}{2 \pi} \int_{-\pi}^{\pi}\left(\frac{\sin \frac{n \lambda}{2}}{\sin \frac{\lambda}{2}}\right)^{2 k} d \lambda .
$$

The Jackson polynomial kernel is $\left|H_{N}(\lambda)\right|^{\alpha}=\left|A_{N} H^{(N)}(\lambda)\right|^{\alpha}$, where $A_{N}=$ $B_{\alpha, N}^{-1 / \alpha}$ with $B_{\alpha, N}=\int_{-\pi}^{\pi}\left|H^{(N)}(\lambda)\right|^{\alpha} d \lambda$. We define $H_{M}(\lambda)$ in the same way.

The following lemma, proved in [6], will be frequently used in the sequel.

Lemma $2.1[6]$. Let

$$
B_{\alpha, N}^{\prime}=\int_{-\pi}^{\pi}\left|\frac{\sin \frac{n \lambda}{2}}{\sin \frac{\lambda}{2}}\right|^{2 k \alpha} d \lambda \quad \text { and } \quad J_{N, \alpha}=\int_{-\pi}^{\pi}|u|^{\gamma}\left|H_{N}(\lambda)\right|^{\alpha} d \lambda,
$$

where $\gamma \in] 0,2]$. Then

$$
B_{\alpha, N}^{\prime} \begin{cases}\geq 2 \pi\left(\frac{2}{\pi}\right)^{2 k \alpha} n^{2 k \alpha-1} & \text { if } 0<\alpha<2 \\ \leq \frac{4 \pi k \alpha}{2 k \alpha-1} n^{2 k \alpha-1} & \text { if } \frac{1}{2 k}<\alpha<2\end{cases}
$$


and

$$
J_{N, \alpha} \leq \begin{cases}\frac{\pi^{\gamma+2 k \alpha}}{2^{2 k \alpha}(\gamma-2 k \alpha+1)} \cdot \frac{1}{n^{2 k \alpha-1}} & \text { if } \frac{1}{2 k}<\alpha<\frac{\gamma+1}{2 k} \\ \frac{2 k \alpha \pi^{\gamma+2 k \alpha}}{2^{2 k \alpha}(\gamma+1)(2 k \alpha-\gamma-1)} \cdot \frac{1}{n^{\gamma}} & \text { if } \frac{\gamma+1}{2 k}<\alpha<2 .\end{cases}
$$

The proof of the following lemma is the same as in the one-dimensional case in [3].

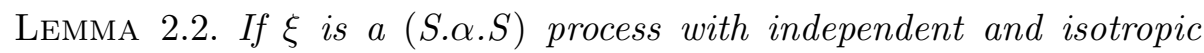
increments, then

$$
\begin{aligned}
\mathbf{E} \exp \left(i \operatorname { R e } \left[\int_{-\pi}^{\pi} \int_{-\pi}^{\pi} f\left(u_{1}, u_{2}\right)\right.\right. & \left.\left.d \xi\left(u_{1}, u_{2}\right)\right]\right) \\
& =\exp \left(-C_{\alpha} \int_{-\pi}^{\pi}\left|f\left(u_{1}, u_{2}\right)\right|^{\alpha} d \mu\left(u_{1}, u_{2}\right)\right)
\end{aligned}
$$

for every $f \in L_{\alpha}(\mu)$, where $C_{\alpha}=(2 \pi \alpha)^{-1} \int_{-\pi}^{\pi}|\cos \theta|^{\alpha} d \theta$.

Now we denote by $\mathcal{A}$ the set of points where there are no atoms:

$$
\begin{aligned}
\mathcal{A}=\left\{\left(\lambda_{1}, \lambda_{2}\right) \in\right]-\pi, \pi\left[^{2}: \lambda_{1} \neq w_{1 j}, \lambda_{2} \neq w_{2 j}\right. \text { and } & \\
& \left.\frac{\lambda_{2}-b_{i}-a_{i} \lambda_{1}}{2 \pi} \notin \mathbb{Z} \text { for } 1 \leq i \leq q^{\prime} \text { and } 1 \leq j \leq q\right\} .
\end{aligned}
$$

In the sequel we choose large $k$ such that $k \alpha>1$ and we consider the following periodogram:

$$
\begin{aligned}
d_{N, M}\left(\lambda_{1}, \lambda_{2}\right)=A_{N} & A_{M} \operatorname{Re}\left[\sum_{n^{\prime}=-k(n-1)}^{k(n-1)} \sum_{m^{\prime}=-k(m-1)}^{k(m-1)} h_{k}\left(\frac{n^{\prime}}{n}\right) h_{k}\left(\frac{m^{\prime}}{m}\right)\right. \\
& \left.\times e^{-i\left(\lambda_{1} n^{\prime}+\lambda_{2} m^{\prime}\right)} X\left(n^{\prime}+k(n-1), m^{\prime}+k(m-1)\right)\right] .
\end{aligned}
$$

Following the study realized in the one-dimensional case by Masry and Cambanis in [11], one can show easily that, for $\left(\lambda_{1}, \lambda_{2}\right) \in \mathcal{A}$,

$$
\lim _{N \rightarrow \infty} \mathbf{E}\left\{\exp \left[i r d_{N, M}\left(\lambda_{1}, \lambda_{2}\right)\right]\right\}=\exp \left[-C_{\alpha}|r|^{\alpha} \phi\left(\lambda_{1}, \lambda_{2}\right)\right] .
$$

We modify this periodogram taking the power $p$, with $0<p<\alpha / 2$, and multiplying by a normalization constant:

$$
\widehat{I}_{N, M}\left(\lambda_{1}, \lambda_{2}\right)=C_{p, \alpha}\left|d_{N, M}\left(\lambda_{1}, \lambda_{2}\right)\right|^{p} .
$$


The normalization constant $C_{p, \alpha}$ is given by

$$
C_{p, \alpha}=\frac{D_{p}}{F_{p, \alpha} C_{\alpha}^{p / \alpha}}
$$

where

$$
D_{p}=\int_{-\infty}^{\infty} \frac{1-\cos u}{|u|^{1+p}} d u \quad \text { and } \quad F_{p, \alpha}=\int_{-\infty}^{\infty} \frac{1-e^{-|u|^{\alpha}}}{|u|^{1+p}} d u, \quad 0<p<\frac{\alpha}{2}
$$

We show that

$$
\begin{aligned}
\mathbf{E} \widehat{I}_{N, M}\left(\lambda_{1}, \lambda_{2}\right) & =\left[\psi_{N, M}\left(\lambda_{1}, \lambda_{2}\right)\right]^{p / \alpha}, \\
\operatorname{Var}\left[\widehat{I}_{N, M}\left(\lambda_{1}, \lambda_{2}\right)\right] & =V_{\alpha, p}\left[\psi_{N, M}\left(\lambda_{1}, \lambda_{2}\right)\right]^{2 p / \alpha},
\end{aligned}
$$

where $V_{\alpha, p}=C_{p, \alpha}^{2} C_{2 p, \alpha}^{-1}-1$ and

$$
\psi_{N, M}\left(\lambda_{1}, \lambda_{2}\right)=I_{N, M}\left(\lambda_{1}, \lambda_{2}\right)+J_{N, M}\left(\lambda_{1}, \lambda_{2}\right)+K_{N, M}\left(\lambda_{1}, \lambda_{2}\right)
$$

with

$$
\begin{aligned}
I_{N, M}\left(\lambda_{1}, \lambda_{2}\right) & =\int_{-\pi}^{\pi} \int_{-\pi}^{\pi}\left|H_{N}\left(\lambda_{1}-u_{1}\right)\right|^{\alpha}\left|H_{M}\left(\lambda_{2}-u_{2}\right)\right|^{\alpha} \phi\left(u_{1}, u_{2}\right) d u_{1} d u_{2}, \\
J_{N, M}\left(\lambda_{1}, \lambda_{2}\right) & =\sum_{j=1}^{q} a_{j}^{\prime}\left|H_{N}\left(\lambda_{1}-w_{1 j}\right)\right|^{\alpha}\left|H_{M}\left(\lambda_{2}-w_{2 j}\right)\right|^{\alpha}, \\
K_{N, M}\left(\lambda_{1}, \lambda_{2}\right) & =\sum_{i=1}^{q^{\prime}} \int_{-\pi}^{\pi}\left|H_{N}\left(\lambda_{1}-v_{1}\right)\right|^{\alpha}\left|H_{M}\left(\lambda_{2}-a_{i} v_{1}-b_{i}\right)\right|^{\alpha} \phi_{i}\left(v_{1}\right) d v_{1} .
\end{aligned}
$$

As in [11] one can show that for $\left(\lambda_{1}, \lambda_{2}\right) \in \mathcal{A}$, the function $\psi_{N, M}\left(\lambda_{1}, \lambda_{2}\right)$ converges to $\phi\left(\lambda_{1}, \lambda_{2}\right)$. Therefore $\widehat{I}_{N, M}\left(\lambda_{1}, \lambda_{2}\right)$ is an asymptotically unbiased estimator of $\left[\phi\left(\lambda_{1}, \lambda_{2}\right)\right]^{p / \alpha}$ but it is not consistent because the variance is proportional to $\left[\phi\left(\lambda_{1}, \lambda_{2}\right)\right]^{2 p / \alpha}$.

3. The smoothed periodogram. In this section, using two spectral windows, we smooth the periodogram $\widehat{I}_{N, M}$ and we obtain consistent estimators of $\left[\phi\left(\lambda_{1}, \lambda_{2}\right)\right]^{p / \alpha}$ at the points $\left(\lambda_{1}, \lambda_{2}\right)$ where there are no atoms. Let $W$ be a nonnegative, even, continuous function vanishing for $|\lambda|>1$, with $\int_{-1}^{1} W(\lambda) d \lambda=1$. The spectral windows $W_{N}, W_{M}$ are defined by

$$
W_{N}(\lambda)=M_{N} W\left(M_{N} \lambda\right) \quad \text { and } \quad W_{M}(\lambda)=L_{M} W\left(L_{M} \lambda\right)
$$

where $M_{N}$ and $L_{M}$ satisfy

$$
\begin{array}{ll}
\lim _{N \rightarrow \infty} M_{N}=\infty, & \lim _{N \rightarrow \infty} \frac{M_{N}}{N}=0, \\
\lim _{M \rightarrow \infty} L_{M}=\infty, & \lim _{M \rightarrow \infty} \frac{L_{M}}{M}=0 .
\end{array}
$$


We consider the following estimator:

$$
f_{N, M}\left(\lambda_{1}, \lambda_{2}\right)=\int_{-\pi}^{\pi} \int_{-\pi}^{\pi} W_{N}\left(\lambda_{1}-u_{1}\right) W_{M}\left(\lambda_{2}-u_{2}\right) \widehat{I}_{N, M}\left(u_{1}, u_{2}\right) d u_{1} d u_{2}
$$

As in [6], for giving the best rate of convergence of this estimator we introduce on $\phi$ two hypotheses $\left(h_{1}\right)$ and $\left(h_{2}\right)$ called regularity hypotheses:

$\left(h_{1}\right) \quad \phi\left(\lambda_{1}-u_{1}, \lambda_{2}-u_{2}\right)=\phi\left(\lambda_{1}, \lambda_{2}\right)+R_{1}\left(\lambda_{1}, \lambda_{2}, u_{1}, u_{2}\right)$

with $\left|R_{1}\left(\lambda_{1}, \lambda_{2}, u_{1}, u_{2}\right)\right| \leq C_{1}\left\|\left(u_{1}, u_{2}\right)\right\|^{\gamma}, \quad$ where $0<\gamma \leq 1$,

$\left(h_{2}\right) \quad \phi\left(\lambda_{1}-u_{1}, \lambda_{2}-u_{2}\right)=\phi\left(\lambda_{1}, \lambda_{2}\right)+\frac{\partial \phi}{\partial x}\left(\lambda_{1}, \lambda_{2}\right) u_{1}+\frac{\partial \phi}{\partial y}\left(\lambda_{1}, \lambda_{2}\right) u_{2}$

$$
+R_{2}\left(\lambda_{1}, \lambda_{2}, u_{1}, u_{2}\right)
$$

with $\left|R_{2}\left(\lambda_{1}, \lambda_{2}, u_{1}, u_{2}\right)\right| \leq C_{2}\left\|\left(u_{1}, u_{2}\right)\right\|^{\gamma}, \quad$ where $1 \leq \gamma<2$,

$C_{1}$ and $C_{2}$ being nonnegative constants.

Theorem 3.1. Let $\lambda_{1}, \lambda_{2} \in \mathcal{A}$. Then:

(i) $f_{N, M}\left(\lambda_{1}, \lambda_{2}\right)$ is an asymptotically unbiased estimator of the quantity $\left[\phi\left(\lambda_{1}, \lambda_{2}\right)\right]^{p / \alpha}$.

(ii) Choosing $k$ so large that $\gamma+1<2 k \alpha$, we have

$\mathbf{E}\left[f_{N, M}\left(\lambda_{1}, \lambda_{2}\right)\right]-\left[\phi\left(\lambda_{1}, \lambda_{2}\right)\right]^{p / \alpha}$

$$
= \begin{cases}O\left(\frac{1}{M_{N}^{\gamma}}+\frac{1}{\left(L_{M}\right)^{\gamma}}\right) & \text { if } \phi \text { satisfies }\left(h_{1}\right), \\ O\left(\frac{1}{M_{N}}+\frac{1}{L_{M}}\right) & \text { if } \phi \text { satisfies }\left(h_{2}\right) .\end{cases}
$$

Pr o of. Using (2.2), we have

$$
\begin{aligned}
\mathbf{E}\left[f_{N, M}\left(\lambda_{1}, \lambda_{2}\right)\right]= & \int_{M_{N}\left(\lambda_{1}-\pi\right)}^{M_{N}\left(\lambda_{1}+\pi\right)} \int_{L_{M}\left(\lambda_{2}-\pi\right)}^{L_{M}\left(\lambda_{2}+\pi\right)} W(u) W(v) \\
& \times\left[\psi_{N, M}\left(\lambda_{1}-u / M_{N}, \lambda_{2}-v / L_{M}\right)\right]^{p / \alpha} d u d v .
\end{aligned}
$$

Since $W$ is vanishing for $|\lambda|>1$ and $p / \alpha<1$, for $N$ and $M$ large enough we obtain

$$
\begin{aligned}
& \left|\mathbf{E}\left[f_{N, M}\left(\lambda_{1}, \lambda_{2}\right)\right]-\left[\phi\left(\lambda_{1}, \lambda_{2}\right)\right]^{p / \alpha}\right| \\
& \leq \int_{-1}^{1} \int_{-1}^{1} W(u) W(v) \\
& \quad \times\left|\psi_{N, M}\left(\lambda_{1}-u / M_{N}, \lambda_{2}-v / L_{M}\right)-\phi\left(\lambda_{1}, \lambda_{2}\right)\right|^{p / \alpha} d u d v
\end{aligned}
$$


Using the facts that $H_{N}$ and $H_{M}$ are $2 \pi$-periodic, $\left|H_{N}\right|^{\alpha}$ and $\left|H_{M}\right|^{\alpha}$ are two kernels, and $\phi$ is uniformly continuous on $[-\pi, \pi]^{2}$, we find that

$$
\lim _{\substack{N \rightarrow \infty \\ M \rightarrow \infty}} I_{N, M}\left(\lambda_{1}-u / M_{N}, \lambda_{2}-v / L_{M}\right)-\phi\left(\lambda_{1}, \lambda_{2}\right)=0 .
$$

On the other hand,

$$
\begin{aligned}
& J_{N, M}\left(\lambda_{1}-u / M_{N}, \lambda_{2}-v / L_{M}\right) \\
& \leq \sum_{j=1}^{q} \frac{a_{j}^{\prime}}{B_{\alpha, N}^{\prime} B_{\alpha, M}^{\prime}} \\
& \quad \times \frac{1}{\left|\sin \left[\frac{1}{2}\left(\lambda_{1}-u / M_{N}-w_{1 j}\right)\right]\right|^{2 k \alpha}\left|\sin \left[\frac{1}{2}\left(\lambda_{2}-v / L_{M}-w_{2 j}\right)\right]\right|^{2 k \alpha}} .
\end{aligned}
$$

Since $\lambda_{1} \neq w_{1 j}$ and $\lambda_{2} \neq w_{2 j}$ for $j \in\{1, \ldots, q\}$, using Lemma 2.1 we obtain

$$
J_{N, M}\left(\lambda_{1}-u / M_{N}, \lambda_{2}-v / L_{M}\right)=O\left(\frac{1}{n^{2 k \alpha-1} m^{2 k \alpha-1}}\right) .
$$

Considering all possible cases, and partitioning the integrals, we show easily (see [13]) that

$$
\begin{aligned}
K_{N, M}\left(\lambda_{1}-u / M_{N}, \lambda_{2}\right. & \left.-v / L_{M}\right) \\
& =O\left(\frac{1}{m^{2 k \alpha-1}}+\frac{1}{n^{2 k \alpha-1}}+\frac{1}{n^{2 k \alpha-1} m^{2 k \alpha-1}}\right) .
\end{aligned}
$$

Using the inequality

$$
\left|x^{r}-y^{r}\right| \leq \frac{r}{2}\left(x^{r-1}+y^{r-1}\right)|x-y|, \quad x, y \in \mathbb{R}^{+*}, r \in[0,1] \cup[2, \infty[,
$$

and the equalities $(3.2),(3.3)$ we can show that

$$
\left[\psi_{N, M}\left(\lambda_{1}-u / M_{N}, \lambda_{2}-v / L_{M}\right)\right]^{p / \alpha-1}+\left[\phi\left(\lambda_{1}, \lambda_{2}\right)\right]^{p / \alpha-1}
$$

is bounded by a constant, for $N$ and $M$ large enough. We get

$$
\begin{aligned}
\left|\mathbf{E}\left[f_{N, M}\left(\lambda_{1}, \lambda_{2}\right)\right]-\left[\phi\left(\lambda_{1}, \lambda_{2}\right)\right]^{p / \alpha}\right| \leq \mathrm{const} \int_{-1}^{1} \int_{-1}^{1} W(u) W(v) \\
\times\left|\psi_{N, M}\left(\lambda_{1}-u / M_{N}, \lambda_{2}-v / L_{M}\right)-\phi\left(\lambda_{1}, \lambda_{2}\right)\right| d u d v .
\end{aligned}
$$

1) If $\phi$ satisfies the hypothesis $\left(h_{1}\right)$ then we have

$$
\begin{aligned}
& \left|\psi_{N, M}\left(\lambda_{1}-u / M_{N}, \lambda_{2}-v / L_{M}\right)-\phi\left(\lambda_{1}, \lambda_{2}\right)\right| \\
& \leq C_{1} \int_{\lambda_{1}-u / M_{N}-\pi}^{\lambda_{1}-u / M_{N}+\pi} \int_{\lambda_{2}-v / L_{M}-\pi}^{\lambda_{2}-v / L_{M}+\pi}\left|H_{N}(t) H_{M}\left(t^{\prime}\right)\right|^{\alpha} \\
& \quad \times\left(\left|u / M_{N}+t\right|+\left|v / L_{M}+t^{\prime}\right|\right)^{\gamma} d t d t^{\prime} \\
& \quad+J_{N, M}\left(\lambda_{1}-u / M_{N}, \lambda_{2}-v / L_{M}\right)+K_{N, M}\left(\lambda_{1}-u / M_{N}, \lambda_{2}-v / L_{M}\right) .
\end{aligned}
$$


Using twice the inequality

$$
|x+y|^{r} \leq \begin{cases}|x|^{r}+|y|^{r} & \text { if } 0<r \leq 1 \\ 2^{r}\left(|x|^{r}+|y|^{r}\right) & \text { if } r \geq 1\end{cases}
$$

we show that

$$
\begin{aligned}
& \frac{1}{\text { const }}\left|\mathbf{E}\left[f_{N, M}\left(\lambda_{1}, \lambda_{2}\right)\right]\left[\phi\left(\lambda_{1}, \lambda_{2}\right)\right]^{p / \alpha}\right| \\
& \leq \frac{C_{1}}{M_{N}^{\gamma}} \int_{-1}^{1} W(u)|u|^{\gamma} d u \\
&+C_{1} \int_{-1}^{1} W(u) \int_{\lambda_{1}-u / M_{N}-\pi}^{\lambda_{1}-u / M_{N}+\pi}\left|H_{N}(t)\right|^{\alpha}|t|^{\gamma} d t d u \\
&+\frac{C_{1}}{L_{M}^{\gamma}} \int_{-1}^{1} W(v)|v|^{\gamma} d v \\
&+C_{1} \int_{-1}^{1} W(v) \int_{-1}^{\lambda_{2}-v / L_{M}+\pi}\left|H_{M}\left(t^{\prime}\right)\right|^{\alpha}\left|t^{\prime}\right|^{\gamma} d t^{\prime} d v \\
&+\int_{-1}^{1} \int_{-1}^{1} W(u) W(v) \\
& \times\left[J_{N, M}\left(\lambda_{1}-u / M_{N}, \lambda_{2}-v / L_{M}\right)\right. \\
&\left.+K_{N, M}\left(\lambda_{1}-u / M_{N}, \lambda_{2}-v / L_{M}\right)\right] d u d v .
\end{aligned}
$$

On the other hand, since $H_{N}$ is an even function, we have

$$
\begin{aligned}
& \quad \int_{\lambda_{1}-u / M_{N}-\pi}^{\lambda_{1}-u / M_{N}+\pi}\left|H_{N}(t)\right|^{\alpha}|t|^{\gamma} d t \\
& \quad \leq \int_{-\pi}^{\pi}\left|H_{N}(t)\right|^{\alpha}|t|^{\gamma} d t+(2 \pi)^{\gamma} \int_{\pi}^{\left|\lambda_{1}\right|+\left|u / M_{N}\right|+\pi}\left|H_{N}(t)\right|^{\alpha}|t|^{\gamma} d t .
\end{aligned}
$$

Using Lemma 2.1, for $N$ large enough we have

$$
\int_{\pi}^{\left|\lambda_{1}\right|+\left|u / M_{N}\right|+\pi}\left|H_{N}(t)\right|^{\alpha}|t|^{\gamma} d t=O\left(T_{N}\left(\lambda_{1}\right)\right),
$$

where

$$
T_{N}\left(\lambda_{1}\right)= \begin{cases}\frac{1}{n^{2 k \alpha-1}} & \text { if } \lambda_{1} \neq 0 \\ \frac{1}{M_{N} n^{2 k \alpha-1}} & \text { if } \lambda_{1}=0\end{cases}
$$


We gather the results (3.2), (3.3) and (3.5) to obtain

$$
\begin{aligned}
\left|\mathbf{E}\left[f_{N, M}\left(\lambda_{1}, \lambda_{2}\right)\right]-\left[\phi\left(\lambda_{1}, \lambda_{2}\right)\right]^{p / \alpha}\right| & \\
= & O\left(T_{N}\left(\lambda_{1}\right)+T_{M}\left(\lambda_{2}\right)+\frac{1}{M_{N}^{\gamma}}+\frac{1}{L_{M}^{\gamma}}\right. \\
& +\frac{1}{n^{\gamma}}+\frac{1}{m^{\gamma}} \\
& \left.+\frac{1}{n^{2 k \alpha-1}}+\frac{1}{m^{2 k \alpha-1}}+\frac{1}{n^{2 k \alpha-1} m^{2 k \alpha-1}}\right) .
\end{aligned}
$$

Since $0<\gamma \leq 1$ and $\gamma+1<2 k \alpha$, it is clear that

$$
\left|\mathbf{E}\left[f_{N, M}\left(\lambda_{1}, \lambda_{2}\right)\right]-\left[\phi\left(\lambda_{1}, \lambda_{2}\right)\right]^{p / \alpha}\right|=O\left(\frac{1}{M_{N}^{\gamma}}+\frac{1}{L_{M}^{\gamma}}\right) .
$$

2) If $\phi$ satisfies the hypothesis $\left(h_{2}\right)$, using the facts that $H_{N}, H_{M}$ are $2 \pi$-periodic kernels, and (3.5), (3.4) we get

$$
\begin{aligned}
\frac{1}{\text { const }} \mid \mathbf{E}\left[f_{N, M}\right. & \left.\left(\lambda_{1}, \lambda_{2}\right)\right]-\left[\phi\left(\lambda_{1}, \lambda_{2}\right)\right]^{p / \alpha} \mid \\
\leq & \left|\frac{\partial \phi}{\partial x}\left(\lambda_{1}, \lambda_{2}\right)\right| \int_{-1}^{1}\left|u / M_{N}\right| W(u) d u \\
& +\left|\frac{\partial \phi}{\partial y}\left(\lambda_{1}, \lambda_{2}\right)\right| T_{M}\left(\lambda_{2}\right) \\
& +\left|\frac{\partial \phi}{\partial x}\left(\lambda_{1}, \lambda_{2}\right)\right| T_{N}\left(\lambda_{1}\right)+\left|\frac{\partial \phi}{\partial y}\left(\lambda_{1}, \lambda_{2}\right)\right| \int_{-1}^{1}\left|v / L_{M}\right| W(v) d v \\
& +2^{\gamma} \frac{C_{2}}{M_{N}^{\gamma}} \int_{-1}^{1}|u|^{\gamma} W(u) d u+2^{\gamma} \frac{C_{2}}{L_{M}^{\gamma}} \int_{-1}^{1}|v|^{\gamma} W(v) d v \\
& +2^{\gamma} C_{2} \int_{\lambda_{1}-u / M_{N}+\pi}^{\lambda_{1}-u / M_{N}-\pi}\left|H_{N}(t)\right|^{\alpha}|t|^{\gamma} d t \\
& +2^{\gamma} C_{2} \int_{\lambda_{2}-v / L_{M}+\pi}\left|H_{M}\left(t^{\prime}\right)\right|^{\alpha}\left|t^{\prime}\right|^{\gamma} d t^{\prime} \\
& +\int_{-1}^{1} \int_{-1}^{1} \int_{2}-v(u) W(v) \\
& \times\left[J_{N, M}\left(\lambda_{1}-u / M_{N}, \lambda_{2}-v / L_{M}\right)\right. \\
& \left.+K_{N, M}\left(\lambda_{1}-u / M_{N}, \lambda_{2}-v / L_{M}\right)\right] d u d v .
\end{aligned}
$$


We use again the results (3.2), (3.3) and (3.5) to obtain

$$
\begin{aligned}
\left|\mathbf{E}\left[f_{N, M}\left(\lambda_{1}, \lambda_{2}\right)\right]-\left[\phi\left(\lambda_{1}, \lambda_{2}\right)\right]^{p / \alpha}\right| & =O\left(\frac{1}{M_{N}}+\frac{1}{L_{M}}+\frac{1}{L_{M}^{\gamma}}+\frac{1}{M_{N}^{\gamma}}+T_{N}\left(\lambda_{1}\right)+T_{M}\left(\lambda_{2}\right)\right. \\
& \left.+\frac{1}{n^{\gamma}}+\frac{1}{m^{\gamma}}+\frac{1}{n^{2 k \alpha-1}}+\frac{1}{m^{2 k \alpha-1}}+\frac{1}{n^{2 k \alpha-1} m^{2 k \alpha-1}}\right) .
\end{aligned}
$$

Since $1<\gamma \leq 2$, we have $1 / k<(\gamma+1) /(2 k)<\alpha$. Thus, we obtain

$$
\left|\mathbf{E}\left[f_{N, M}\left(\lambda_{1}, \lambda_{2}\right)\right]-\left[\phi\left(\lambda_{1}, \lambda_{2}\right)\right]^{p / \alpha}\right|=O\left(\frac{1}{M_{N}}+\frac{1}{L_{M}}\right) .
$$

Now we show the following lemma which will be used in the sequel.

Lemma 3.2. Let $\left(\lambda_{1,1}, \lambda_{2,1}\right)$ and $\left(\lambda_{1,2}, \lambda_{2,2}\right)$ belong to $\mathcal{A}$. Write

$$
\begin{aligned}
& Q_{N, M}\left(\lambda_{1,1}, \lambda_{1,2}, \lambda_{2,1}, \lambda_{2,2}\right) \\
& =\int_{-\pi}^{\pi} \int_{-\pi}^{\pi} \mid H_{N}\left(\lambda_{1,1}-v_{1}\right) H_{M}\left(\lambda_{2,1}-v_{2}\right) \\
& \quad \times\left. H_{N}\left(\lambda_{1,2}-v_{1}\right) H_{M}\left(\lambda_{2,2}-v_{2}\right)\right|^{\alpha / 2} d \mu\left(v_{1}, v_{2}\right) .
\end{aligned}
$$

Then

$$
\begin{aligned}
& Q_{N, M}\left(\lambda_{1,1}, \lambda_{1,2}, \lambda_{2,1}, \lambda_{2,2}\right) \\
& \leq O\left(\frac{1}{n^{2 k \alpha-1}}+\frac{1}{m^{2 k \alpha-1}}+\frac{1}{n^{2 k \alpha-1} m^{2 k \alpha-1}}\right) \\
& \quad+\left(\frac{\sup (\phi)}{\pi^{2}}\left(\frac{\pi}{2}\right)^{4 k \alpha} \frac{1}{(n m)^{2 k \alpha-1}}\right) \Re\left(\delta, \lambda_{1,2}, \lambda_{1,1}\right) \Re\left(\delta^{\prime}, \lambda_{2,2}, \lambda_{2,1}\right),
\end{aligned}
$$

where the function $\Re$ is defined by

$$
\Re(x, y, z)=\frac{\pi}{\left(\sin \frac{x}{2}\right)^{2 k \alpha}}+\frac{2 x}{\inf \left[\left(\sin \frac{|y-z|+x}{2}\right)^{k \alpha},\left(\sin \frac{|y-z|}{4}\right)^{k \alpha}\right]},
$$

with two real numbers $\delta, \delta^{\prime}$ satisfying

$$
\begin{aligned}
& 0<\delta<\inf \left[\pi-\lambda_{1,2} ; \pi+\lambda_{1,1} ;\left|\lambda_{2,1}-\lambda_{1,1}\right| / 2\right], \\
& 0<\delta^{\prime}<\inf \left[\pi-\lambda_{2,2} ; \pi+\lambda_{2,1} ;\left|\lambda_{2,2}-\lambda_{2,1}\right| / 2\right] .
\end{aligned}
$$

Proof. First using the expression of $d \mu$ in (1.1) and the inequality $x^{\alpha / 2} y^{\alpha / 2} \leq \frac{1}{2}\left(x^{\alpha}+y^{\alpha}\right)$, we obtain

$$
\begin{aligned}
Q_{N, M}\left(\lambda_{1}, \lambda_{2}, x_{1}, x_{2}\right) \leq & B+\frac{1}{2} J_{N, M}\left(\lambda_{1,1}, \lambda_{2,1}\right) \frac{1}{2} J_{N, M}\left(\lambda_{1,2}, \lambda_{2,2}\right) \\
& +\frac{1}{2} K_{N, M}\left(\lambda_{1,1}, \lambda_{2,1}\right)+\frac{1}{2} K_{N, M}\left(\lambda_{1,2}, \lambda_{2,2}\right)
\end{aligned}
$$


where

$$
\begin{aligned}
B= & \int_{-\pi}^{\pi} \int_{-\pi}^{\pi} \mid H_{N}\left(\lambda_{1,1}-v_{1}\right) H_{M}\left(\lambda_{2,1}-v_{2}\right) \\
& \times\left. H_{N}\left(\lambda_{1,2}-v_{1}\right) H_{M}\left(\lambda_{2,2}-v_{2}\right)\right|^{\alpha / 2} \phi\left(v_{1}, v_{2}\right) d v_{1} d v_{2} .
\end{aligned}
$$

Since $\phi$ is bounded on $[-\pi, \pi]^{2}$, we have

$$
\begin{aligned}
B \leq & \sup (\phi)\left(\int_{-\pi}^{\pi}\left|H_{N}\left(\lambda_{1,1}-v_{1}\right) H_{N}\left(\lambda_{1,2}-v_{1}\right)\right|^{\alpha / 2} d v_{1}\right) \\
& \times\left(\int_{-\pi}^{\pi}\left|H_{M}\left(\lambda_{2,2}-v_{2}\right) H_{M}\left(\lambda_{2,1}-v_{2}\right)\right|^{\alpha / 2} d v_{2}\right) .
\end{aligned}
$$

We split the first integral into five integrals: over a neighbourhood of $\lambda_{1,1}$, a neighbourhood of $\lambda_{1,2}$ and the remainders:

$$
\begin{aligned}
\int_{-\pi}^{\pi} \mid H_{N}\left(\lambda_{1,1}-\right. & \left.v_{1}\right)\left.H_{N}\left(\lambda_{1,2}-v_{1}\right)\right|^{\alpha / 2} d v_{1} \\
= & \frac{1}{B_{\alpha, N}^{\prime}}\left(\int_{-\pi}^{\lambda_{1,1}-\delta}+\int_{\lambda_{1,1}-\delta}^{\lambda_{1,1}+\delta}+\int_{\lambda_{1,1}+\delta}^{\lambda_{1,2}-\delta}+\int_{\lambda_{1,2}-\delta}^{\lambda_{1,2}+\delta}\right. \\
& \left.+\int_{\lambda_{1,2}+\delta}^{\pi}\right)\left|\frac{\sin \left[\frac{n}{2}\left(\lambda_{1,1}-w\right)\right]}{\sin \frac{\lambda_{1,1}-w}{2}} \cdot \frac{\sin \left[\frac{n}{2}\left(\lambda_{1,2}-w\right)\right]}{\sin \frac{\lambda_{1,2}-w}{2}}\right|^{k \alpha} d w
\end{aligned}
$$

The first, third and last integrals are respectively bounded by

$$
\frac{1}{B_{\alpha, N}^{\prime}} \cdot \frac{\lambda_{1,1}-\delta+\pi}{\left(\sin \frac{\delta}{2}\right)^{2 k \alpha}}, \quad \frac{1}{B_{\alpha, N}^{\prime}} \cdot \frac{\lambda_{1,2}-\lambda_{1,1}-2 \delta}{\left(\sin \frac{\delta}{2}\right)^{2 k \alpha}}
$$

and

$$
\frac{1}{B_{\alpha, N}^{\prime}} \cdot \frac{\pi-\lambda_{1,2}-\delta}{\left(\sin \frac{\delta}{2}\right)^{2 k \alpha}}
$$

Using the inequality $\sin (n x) \leq n \sin x$, we obtain

$$
\begin{aligned}
\frac{1}{B_{\alpha, N}^{\prime}} \int_{\lambda_{1,1}-\delta}^{\lambda_{1,1}+\delta}\left|\frac{\sin \left[\frac{n}{2}\left(\lambda_{1,1}-w\right)\right]}{\sin \frac{\lambda_{1,1}-w}{2}} \frac{\sin \left[\frac{n}{2}\left(\lambda_{1,2}-w\right)\right]}{\sin \frac{\lambda_{1,2}-w}{2}}\right|^{k \alpha} d w \\
\leq \frac{1}{B_{\alpha, N}^{\prime}} \cdot \frac{2 \delta n^{k \alpha}}{\inf \left[\left(\sin \frac{\left|\lambda_{1,2}-\lambda_{1,1}\right|+\delta}{2}\right)^{k \alpha},\left(\sin \frac{\left|\lambda_{1,2}-\lambda_{1,1}\right|}{4}\right)^{k \alpha}\right]}
\end{aligned}
$$

Similarly, the remaining integral is bounded by the same quantity. Thus 
using Lemma 2.1, we have

$$
\begin{aligned}
\int_{-\pi}^{\pi} \mid H_{N} & \left.\left(\lambda_{1,1}-v_{1}\right) H_{N}\left(\lambda_{1,2}-v_{1}\right)\right|^{\alpha / 2} d v_{1} \\
= & \frac{1}{2 \pi}\left(\frac{\pi}{2}\right)^{2 k \alpha} \frac{1}{n^{2 k \alpha-1}} \\
& \times\left[\frac{2 \pi}{\left(\sin \frac{\delta}{2}\right)^{2 k \alpha}}+\frac{4 \delta n^{k \alpha}}{\inf \left[\left(\sin \frac{\lambda_{1,2}-\lambda_{1,1}+\delta}{2}\right)^{k \alpha}, \sin \left(\frac{\lambda_{1,2}-\lambda_{1,1}}{4}\right)^{k \alpha}\right]}\right]
\end{aligned}
$$

Just as before, we have

$$
\begin{aligned}
\int_{-\pi}^{\pi}\left|H_{M}\left(\lambda_{2,1}-v_{2}\right) H_{M}\left(\lambda_{2,2}-v_{2}\right)\right|^{\alpha / 2} d v_{2} & \\
\leq & \frac{1}{\pi}\left(\frac{\pi}{2}\right)^{2 k \alpha} \frac{1}{m^{2 k \alpha-1}} \Re\left(\delta^{\prime}, \lambda_{2,2}, \lambda_{2,1}\right) .
\end{aligned}
$$

Equalities (3.2) and (3.3) give the result of this lemma.

Theorem 3.3. Let $\left(\lambda_{1}, \lambda_{2}\right) \in \mathcal{A}$ be such that $\phi\left(\lambda_{1}, \lambda_{2}\right) \neq 0$. Then:

(i) $\operatorname{Var}\left[f_{N, M}\left(\lambda_{1}, \lambda_{2}\right)\right]$ converges to zero.

(ii) If $\phi$ satisfies $\left(h_{1}\right)$ or $\left(h_{2}\right)$, and $M_{N}=n^{c}$ and $L_{M}=m^{c^{\prime}}$, where $c$ and $c^{\prime}$ are two real numbers satisfying

$$
\inf \left(\frac{2 k^{2} \alpha^{2}+1}{6 \alpha^{2} k^{2}}, \frac{k \alpha+2}{3(k \alpha+1)}\right)<c, c^{\prime}<\frac{1}{2},
$$

then

$$
\operatorname{Var}\left[f_{N, M}\left(\lambda_{1}, \lambda_{2}\right)\right]=O\left(\frac{1}{n^{2(1-2 c)}}+\frac{1}{m^{2\left(1-2 c^{\prime}\right)}}\right) .
$$

Proof. When $\phi\left(\lambda_{1}, \lambda_{2}\right)=0$ we do not need to smooth $\widehat{I}_{N, M}$, since its variance tends to zero. We have

$\operatorname{Var}\left[f_{N, M}\left(\lambda_{1}, \lambda_{2}\right)\right]$

$$
\begin{aligned}
= & \int_{-\pi}^{\pi} \int_{-\pi}^{\pi} \int_{-\pi}^{\pi} \int_{-\pi}^{\pi} W_{N}\left(\lambda_{1}-u_{1}\right) W_{M}\left(\lambda_{2}-u_{2}\right) W_{N}\left(\lambda_{1}-u_{1}^{\prime}\right) W_{M}\left(\lambda_{2}-u_{2}^{\prime}\right) \\
& \times \operatorname{Cov}\left[\widehat{I}_{N, M}\left(u_{1}, u_{2}\right), \widehat{I}_{N, M}\left(u_{1}^{\prime}, u_{2}^{\prime}\right)\right] d u_{1} d u_{2} d u_{1}^{\prime} d u_{2}^{\prime} .
\end{aligned}
$$

Putting

$$
\begin{array}{ll}
x_{1}=M_{N}\left(\lambda_{1}-u_{1}\right), & x_{2}=L_{M}\left(\lambda_{2}-u_{2}\right), \\
x_{1}^{\prime}=M_{N}\left(\lambda_{1}-u_{1}^{\prime}\right), & x_{2}^{\prime}=L_{M}\left(\lambda_{2}-u_{2}^{\prime}\right) .
\end{array}
$$

and using the fact that $W$ is vanishing for $|\lambda|>1$, for $N$ and $M$ large enough we have 
$\operatorname{Var}\left[f_{N, M}\left(\lambda_{1}, \lambda_{2}\right)\right]$

$$
\begin{aligned}
= & \int_{-1}^{1} \int_{-1}^{1} \int_{-1}^{1} \int_{-1}^{1} W\left(x_{1}\right) W\left(x_{2}\right) W\left(x_{1}^{\prime}\right) W\left(x_{2}^{\prime}\right) \\
& \times \operatorname{Cov}\left[\widehat{I}_{N, M}\left(\lambda_{1}-x_{1} / M_{N}, \lambda_{2}-x_{2} / L_{M}\right),\right. \\
& \left.\widehat{I}_{N, M}\left(\lambda_{1}-x_{1}^{\prime} / M_{N}, \lambda_{2}-x_{2}^{\prime} / L_{M}\right)\right] d x_{1} d x_{2} d x_{1}^{\prime} d x_{2}^{\prime} .
\end{aligned}
$$

We define

$$
\begin{aligned}
& \mathcal{L}_{1}=\left\{\left(x_{1}, x_{1}^{\prime}\right) \in[-1,1]^{2}:\left|x_{1}-x_{1}^{\prime}\right|>\sigma_{N}\right\}, \\
& \mathcal{L}_{2}=\left\{\left(x_{2}, x_{2}^{\prime}\right) \in[-1,1]^{2}:\left|x_{2}-x_{2}^{\prime}\right|>\sigma_{M}^{\prime}\right\}, \\
& \mathcal{L}_{3}=\left\{\left(x_{1}, x_{1}^{\prime}, x_{2}, x_{2}^{\prime}\right) \in[-1,1]^{4}:\left|x_{1}-x_{1}^{\prime}\right| \leq \sigma_{N} \text { or }\left|x_{2}-x_{2}^{\prime}\right| \leq \sigma_{M}^{\prime}\right\},
\end{aligned}
$$

where $\sigma_{N}, \sigma_{M}^{\prime}$ are two nonnegative real sequences converging to 0 . We split the last integral into the integral over $\mathcal{L}_{3}$ and the integral over $\mathcal{L}_{1}$ and $\mathcal{L}_{2}$ :

$$
\operatorname{Var}\left[f_{N, M}\left(\lambda_{1}, \lambda_{2}\right)\right]=\iiint_{\mathcal{L}_{3}} \int+\iint_{\mathcal{L}_{1}} \iint_{\mathcal{L}_{2}}=: J_{1}+J_{2} .
$$

Using (2.3) and the uniform (in $\left.x_{1}, x_{2}\right)$ convergence of $\psi_{N, M}\left(\lambda_{1}-x_{1} / M_{N}\right.$, $\left.\lambda_{2}-x_{2} / L_{M}\right)$ to $\phi\left(\lambda_{1}, \lambda_{2}\right)$, we obtain

$$
\begin{aligned}
J_{1} \leq \mathrm{const}\left[\int_{\left|x_{2}-x_{2}^{\prime}\right| \leq \sigma_{M}^{\prime}} W\left(x_{2}\right) W\left(x_{2}^{\prime}\right) d x_{2} d x_{2}^{\prime}\right. \\
\left.+\iint_{\left|x_{1}-x_{1}^{\prime}\right| \leq \sigma_{N}} W\left(x_{1}\right) W\left(x_{1}^{\prime}\right) d x_{1} d x_{1}^{\prime}\right] .
\end{aligned}
$$

Thus, $J_{1} \leq \operatorname{const}[\sup (W)]^{2}\left[\sigma_{N}+\sigma_{M}^{\prime}\right]$. Hence $J_{1}$ tends to zero.

It remains to show that $J_{2}$ tends to zero. First we define for simplicity

$$
\begin{array}{ll}
\lambda_{1,1}=\lambda_{1}-x_{1} / M_{N}, & \lambda_{1,2}=\lambda_{1}-x_{1}^{\prime} / M_{N}, \\
\lambda_{2,1}=\lambda_{2}-x_{2} / L_{M}, & \lambda_{2,2}=\lambda_{2}-x_{2}^{\prime} / L_{M},
\end{array}
$$

and

$C\left(\lambda_{1}, \lambda_{2}\right)$

$=\operatorname{Cov}\left[\widehat{I}_{N, M}\left(\lambda_{1}-x_{1} / M_{N}, \lambda_{2}-x_{2} / L_{M}\right), \widehat{I}_{N, M}\left(\lambda_{1}-x_{1}^{\prime} / M_{N}, \lambda_{2}-x_{2}^{\prime} / L_{M}\right)\right]$.

We use the equality

$$
|x|^{p}=D_{p}^{-1} \int_{-\infty}^{\infty} \frac{1-\cos (x u)}{|u|^{1+p}} d u=D_{p}^{-1} \operatorname{Re} \int_{-\infty}^{\infty} \frac{1-e^{i x u}}{|u|^{1+p}} d u
$$

for all real $x$ and $p \in] 0,2[$. We have

$$
\widehat{I}_{N, M}\left(\lambda_{1}, \lambda_{2}\right)=\frac{1}{F_{p, \alpha} C_{\alpha}^{p / \alpha}} \operatorname{Re} \int_{-\infty}^{\infty} \frac{1-e^{i u d_{N, M}\left(\lambda_{1}, \lambda_{2}\right)}}{|u|^{1+p}} d u .
$$


From (2.1) we obtain

$$
\mathbf{E} \widehat{I}_{N, M}\left(\lambda_{1}, \lambda_{2}\right)=\frac{1}{F_{p, \alpha} C_{\alpha}^{p / \alpha}} \int_{-\infty}^{\infty} \frac{1-\exp \left\{-C_{\alpha}|u|^{\alpha} \psi_{N, M}\left(\lambda_{1}, \lambda_{2}\right)\right\}}{|u|^{1+p}} d u
$$

By using (3.6) and (3.7) we show easily that

$$
\begin{aligned}
C\left(\lambda_{1}, \lambda_{2}\right)= & F_{p, \alpha}^{-2} C_{\alpha}^{-2 p / \alpha} \int_{-\infty}^{\infty} \int_{-\infty}^{\infty}\left(\mathbf{E}\left[\prod_{k=1}^{2} \cos \left(u_{k} d_{N, M}\left(\lambda_{1, k}, \lambda_{2, k}\right)\right)\right]\right. \\
& \left.-\exp \left\{-C_{\alpha} \sum_{k=1}^{2}\left|u_{k}\right|^{\alpha} \psi_{N, M}\left(\lambda_{1, k}, \lambda_{2, k}\right)\right\}\right) \frac{d u_{1} d u_{2}}{\left|u_{1} u_{2}\right|^{1+p}}
\end{aligned}
$$

From the equality $2 \cos x \cos y=\cos (x+y)+\cos (x-y)$, we have

$$
\begin{aligned}
\mathbf{E}\left[\prod_{k=1}^{2} u_{k} d_{N, M}\left(\lambda_{1, k}, \lambda_{2, k}\right)\right] & \\
= & \frac{1}{2} \exp \left[-C_{\alpha} \int\left|\sum_{k=1}^{2} u_{k} H_{N}\left(\lambda_{1, k}-v_{1}\right) H_{M}\left(\lambda_{2, k}-v_{2}\right)\right|^{\alpha} d \mu\left(v_{1}, v_{2}\right)\right] \\
& +\frac{1}{2} \exp \left[-C_{\alpha} \int \mid \sum_{k=1}^{2}(-1)^{k-1} u_{k} H_{N}\left(\lambda_{1, k}-v_{1}\right)\right. \\
& \left.\times\left. H_{M}\left(\lambda_{2, k}-v_{2}\right)\right|^{\alpha} d \mu\left(v_{1}, v_{2}\right)\right] .
\end{aligned}
$$

Substituting this expression in $C\left(\lambda_{1}, \lambda_{2}\right)$ and changing the variable $u_{2}$ to $-u_{2}$ in the second term, we obtain

$$
C\left(\lambda_{1}, \lambda_{2}\right)=F_{p, \alpha}^{-2} C_{\alpha}^{-2 p / \alpha} \int_{-\infty}^{\infty} \int_{-\infty}^{\infty}\left(e^{-K}-e^{-K^{\prime}}\right) \frac{d u_{1} d u_{2}}{\left|u_{1} u_{2}\right|^{1+p}},
$$

where

$$
\begin{aligned}
K & =C_{\alpha} \int_{-\pi}^{\pi} \int_{-\pi}^{\pi}\left|\sum_{k=1}^{2} u_{k} H_{N}\left(\lambda_{1, k}-v_{1}\right) H_{M}\left(\lambda_{2, k}-v_{2}\right)\right|^{\alpha} d \mu\left(v_{1}, v_{2}\right) \\
K^{\prime} & =C_{\alpha} \sum_{k=1}^{2}\left|u_{k}\right|^{\alpha} \int_{-\pi}^{\pi} \int_{-\pi}^{\pi}\left|H_{N}\left(\lambda_{1, k}-v_{1}\right) H_{M}\left(\lambda_{2, k}-v_{2}\right)\right|^{\alpha} d \mu\left(v_{1}, v_{2}\right) \\
& =C_{\alpha} \sum_{k=1}^{2}\left|u_{k}\right|^{\alpha} \psi_{N, M}\left(\lambda_{1, k}, \lambda_{2, k}\right) .
\end{aligned}
$$


Since $K, K^{\prime}>0$, we have

$$
\left|e^{-K}-e^{-K^{\prime}}\right| \leq\left|K-K^{\prime}\right| e^{\left|K-K^{\prime}\right|-K^{\prime}}
$$

Using the inequality

$$
\| x+\left.y\right|^{\alpha}-|x|^{\alpha}-\left.|y|^{\alpha}|\leq 2| x y\right|^{\alpha / 2}, \quad x, y \in \mathbb{R} \text { and } 1 \leq \alpha \leq 2,
$$

we obtain

$$
\left|K-K^{\prime}\right| \leq 2 C_{\alpha}\left|u_{1} u_{2}\right|^{\alpha / 2} Q_{N, M}\left(\lambda_{1,1}, \lambda_{1,2}, \lambda_{2,1}, \lambda_{2,2}\right) .
$$

Therefore,

$C\left(\lambda_{1}, \lambda_{2}\right)$

$\leq F_{p, \alpha}^{-2} C_{\alpha}^{-2 p / \alpha} 2 C_{\alpha} Q_{N, M}\left(\lambda_{1,1}, \lambda_{1,2}, \lambda_{2,1}, \lambda_{2,2}\right) \int_{-\infty}^{\infty} \int_{-\infty}^{\infty} \frac{e^{\left|K-K^{\prime}\right|-K^{\prime}}}{\left|u_{1} u_{2}\right|^{1+p-\alpha / 2}} d u_{1} d u_{2}$.

Now we have

$$
\begin{aligned}
\mid K & -K^{\prime} \mid-K^{\prime} \\
& \leq-C_{\alpha} \sum_{k=1}^{2}\left|u_{k}\right|^{\alpha}\left[\psi_{N, M}\left(\lambda_{1, k}, \lambda_{2, k}\right)-Q_{N, M}\left(\lambda_{1,1}, \lambda_{1,2}, \lambda_{2,1}, \lambda_{2,2}\right)\right] .
\end{aligned}
$$

Let $\delta(N), \delta^{\prime}(M)$ be two real numbers depending respectively on $N$ and $M$ such that

$$
\begin{aligned}
& 0<\delta(N)<\inf \left[\left|\lambda_{1,1}-\lambda_{1,2}\right| / 2 ; \pi-\lambda_{1,2} ; \pi+\lambda_{1,1}\right], \\
& 0<\delta^{\prime}(M)<\inf \left[\left|\lambda_{2,1}-\lambda_{2,2}\right| / 2 ; \pi-\lambda_{2,2} ; \pi+\lambda_{2,1}\right] .
\end{aligned}
$$

Moreover, we suppose that

$$
\lim _{N \rightarrow \infty} \frac{\delta(N) M_{N}}{\sigma_{N}}=0, \quad \lim _{M \rightarrow \infty} \frac{\delta^{\prime}(M) L_{M}}{\sigma_{M}^{\prime}}=0 .
$$

Using Lemma 3.2 and the inequality

$$
\sin (x / 2) \geq x / \pi, \quad 0 \leq x \leq \pi,
$$

we obtain

$$
\begin{aligned}
\int_{-\pi}^{\pi} \mid H_{N}\left(\lambda_{1,1}-v\right) & \left.H_{N}\left(\lambda_{1,2}-v\right)\right|^{\alpha / 2} d v \\
\leq & \frac{1}{\pi}\left(\frac{\pi}{2}\right)^{2 k \alpha}\left[\frac{\pi^{2 k \alpha+1}}{n^{2 k \alpha-1} \delta(N)^{2 k \alpha}}+\frac{2 \delta(N)(2 \pi)^{k \alpha}}{n^{k \alpha-1}\left(\sigma_{N} / M_{N}\right)^{k \alpha}}\right] .
\end{aligned}
$$

We choose $\delta(N)=n^{-\beta}, \delta^{\prime}(M)=m^{-\beta^{\prime}}$ with $\beta>0$ and $\beta^{\prime}>0$. In order to have 


$$
\lim _{N \rightarrow \infty} \int_{-\pi}^{\pi}\left|H_{N}\left(\lambda_{1,1}-v\right) H_{N}\left(\lambda_{1,2}-v\right)\right|^{\alpha / 2} d v=0,
$$

it is sufficient that

$$
\lim _{N \rightarrow \infty} \frac{n^{2 k \alpha \beta}}{n^{2 k \alpha-1}}=0, \quad \lim _{N \rightarrow \infty} \frac{1}{n^{\beta+k \alpha-1}\left(\sigma_{N} / M_{N}\right)^{\alpha k}}=0,
$$

and

$$
\lim _{M \rightarrow \infty} \frac{m^{2 k \alpha \beta^{\prime}}}{m^{2 k \alpha-1}}=0, \quad \lim _{M \rightarrow \infty} \frac{1}{m^{\beta^{\prime}+k \alpha-1}\left(\sigma_{M}^{\prime} / L_{M}\right)^{\alpha k}}=0 .
$$

Suppose for a moment that conditions (3.9), (3.11) and (3.12) are fulfilled; thus $Q_{N, M}\left(\lambda_{1,1} ; \lambda_{1,2} ; \lambda_{2,1} ; \lambda_{2,2}\right)$ converges to zero. We write

$$
\Delta_{N, M, k}=\psi_{N, M}\left(\lambda_{1, k}, \lambda_{2, k}\right)-Q_{N, M}\left(\lambda_{1,1} ; \lambda_{1,2} ; \lambda_{2,1} ; \lambda_{2,2}\right) .
$$

Then

$$
\begin{aligned}
& \int_{-\infty}^{\infty} \int_{-\infty}^{\infty} \frac{e^{\left|K-K^{\prime}\right|-K^{\prime}}}{\left|u_{1} u_{2}\right|^{1+p-\alpha / 2}} d u_{1} d u_{2} \\
& \leq 4 \prod_{k=1}^{2} \int_{0}^{\infty} \exp \left(-C_{\alpha}\left|u_{k}\right|^{\alpha} \Delta_{N, M, k}\right) \frac{d u_{k}}{\left|u_{k}\right|^{1+p-\alpha / 2}}
\end{aligned}
$$

Putting $v=u_{k}\left(\Delta_{N, M, k}\right)^{1 / \alpha}$, one has

(3.13) $C\left(\lambda_{1}, \lambda_{2}\right)$

$$
\leq \mathrm{const} \frac{Q_{N, M}\left(\lambda_{1,1} ; \lambda_{1,2} ; \lambda_{2,1} ; \lambda_{2,2}\right)}{\left[\Delta_{N, M, 1} \Delta_{N, M, 2}\right]^{1 / 2-p / \alpha}}\left(\int_{-\infty}^{\infty} \frac{e^{-C_{\alpha}|v|^{\alpha}}}{|v|^{1+p-\alpha / 2}} d v\right)^{2} .
$$

Since $\psi_{N, M}\left(\lambda_{1, k}, \lambda_{2, k}\right)$ converges to $\phi\left(\lambda_{1}, \lambda_{2}\right)$, it follows that $\Delta_{N, M, k}$ converges to $\phi\left(\lambda_{1}, \lambda_{2}\right)$. Thus $J_{2}$ tends to zero.

Now we study the rate of convergence of $J_{2}$. From (3.13) we have $J_{2}=$ $O\left(S_{N, M}\left(\lambda_{1}, \lambda_{2}\right)\right)$, where

$$
\begin{aligned}
S_{N, M}\left(\lambda_{1}, \lambda_{2}\right)= & \int_{-1}^{1} \int_{-1}^{1} \int_{-1}^{1} \int_{-1}^{1} W\left(x_{1}\right) W\left(x_{1}^{\prime}\right) W\left(x_{2}\right) W\left(x_{2}^{\prime}\right) \\
& \times Q_{N, M}\left(\lambda_{1,1} ; \lambda_{1,2} ; \lambda_{2,1} ; \lambda_{2,2}\right) d x_{1} d x_{1}^{\prime} d x_{2} d x_{2}^{\prime} .
\end{aligned}
$$

Using (1.1), (3.2) and (3.3) we get

$$
S_{N, M}\left(\lambda_{1}, \lambda_{2}\right) \leq S_{N, M}^{(1)}\left(\lambda_{1}, \lambda_{2}\right)+O\left(\frac{1}{n^{2 k \alpha-1}}+\frac{1}{m^{2 k \alpha-1}}+\frac{1}{n^{2 k \alpha-1} m^{2 k \alpha-1}}\right),
$$


where

$$
\begin{aligned}
& S_{N, M}^{(1)}\left(\lambda_{1}, \lambda_{2}\right) \\
& =\int_{-\pi}^{\pi} \int_{-\pi}^{\pi} \phi\left(v_{1}, v_{2}\right) \\
& \quad \times\left(\int_{-1}^{1} \int_{-1}^{1} W\left(x_{1}\right) W\left(x_{1}^{\prime}\right)\left|H_{N}\left(\lambda_{1,1}-v_{1}\right) H_{N}\left(\lambda_{1,2}-v_{1}\right)\right|^{\alpha / 2} d x_{1} d x_{1}^{\prime}\right) \\
& \quad \times\left(\int_{-1}^{1} \int_{-1}^{1} W\left(x_{2}\right) W\left(x_{2}^{\prime}\right)\right. \\
& \left.\quad \times\left|H_{M}\left(\lambda_{2,1}-v_{2}\right) H_{M}\left(\lambda_{2,2}-v_{2}\right)\right|^{\alpha / 2} d x_{2} d x_{2}^{\prime}\right) d v_{1} d v_{2} .
\end{aligned}
$$

We have

$$
\begin{aligned}
S_{N, M}^{(1)}\left(\lambda_{1}, \lambda_{2}\right) \leq & \int_{-\pi}^{\pi} \int_{-\pi}^{\pi} \phi\left(v_{1}, v_{2}\right)\left(\int_{-\pi}^{\pi} W_{N}\left(u_{1}\right)\left|H_{N}\left(\lambda_{1}-u_{1}-v_{1}\right)\right|^{\alpha / 2} d u_{1}\right)^{2} \\
& \times\left(\int_{-\pi}^{\pi} W_{M}\left(u_{2}\right)\left|H_{M}\left(\lambda_{2}-u_{2}-v_{2}\right)\right|^{\alpha / 2} d u_{2}\right)^{2} d v_{1} d v_{2} .
\end{aligned}
$$

Changing the variable, we obtain

$$
S_{N, M}^{(1)}\left(\lambda_{1}, \lambda_{2}\right) \leq \int_{-2 \pi}^{2 \pi} \int_{-2 \pi}^{2 \pi} \phi\left(\lambda_{1}-w_{1}, \lambda_{2}-w_{2}\right)\left[G_{N}\left(w_{1}\right)\right]^{2}\left[G_{M}\left(w_{2}\right)\right]^{2} d w_{1} d w_{2},
$$

where

$$
\begin{aligned}
G_{N}\left(w_{1}\right) & =\int_{w_{1}-\pi}^{w_{1}+\pi} W_{N}\left(w_{1}-t_{1}\right)\left|H_{N}\left(t_{1}\right)\right|^{\alpha / 2} d t_{1}, \\
G_{M}\left(w_{2}\right) & =\int_{w_{2}-\pi}^{w_{2}+\pi} W_{M}\left(w_{2}-t_{2}\right)\left|H_{M}\left(t_{2}\right)\right|^{\alpha / 2} d t_{2} .
\end{aligned}
$$

Now,

$$
\begin{aligned}
G_{N}\left(w_{1}\right) & \leq \frac{M_{N} \sup (W)}{\left(B_{\alpha, N}^{\prime}\right)^{1 / 2}} \int_{-3 \pi}^{3 \pi}\left|\frac{\sin \frac{n t}{2}}{\sin \frac{t}{2}}\right|^{k \alpha} d t \\
& \leq \frac{6 M_{N} \sup (W)}{\left(B_{\alpha, N}^{\prime}\right)^{1 / 2}} \int_{0}^{\pi}\left|\frac{\sin \frac{n t}{2}}{\sin \frac{t}{2}}\right|^{k \alpha} d t \\
& \leq \frac{6 M_{N} \sup (W) n^{k \alpha}}{\left(B_{\alpha, N}^{\prime}\right)^{1 / 2}} \int_{0}^{\pi / n} d t_{1}+\frac{6 M_{N} \sup (W)}{\left(B_{\alpha, N}^{\prime}\right)^{1 / 2}} \int_{\pi / n}^{\pi}\left(\frac{\pi}{t_{1}}\right)^{k \alpha} d t_{1} .
\end{aligned}
$$


Thus from Lemma 2.1 we get

$$
G_{N}\left(w_{1}\right)=O\left(\frac{M_{N}}{n^{1 / 2}}\right) \quad \text { and } \quad G_{M}\left(w_{2}\right)=O\left(\frac{L_{M}}{m^{1 / 2}}\right) .
$$

Thus we have

$$
J_{2}=O\left(\frac{1}{n^{2 k \alpha-1}}+\frac{1}{m^{2 k \alpha-1}}+\frac{1}{n^{2 k \alpha-1} m^{2 k \alpha-1}}+\frac{M_{N}^{2}}{n} \cdot \frac{L_{M}^{2}}{m}\right) .
$$

Since $k \alpha>1$, it follows that

$$
J_{2}=O\left(\frac{M_{N}^{4}}{n^{2}}+\frac{L_{M}^{4}}{m^{2}}\right) .
$$

Using the evaluation obtained for $J_{1}$, we have

$$
\operatorname{Var}\left[f_{N, M}\left(\lambda_{1}, \lambda_{2}\right)\right]=O\left(\sigma_{N}+\sigma_{N}^{\prime}+\frac{M_{N}^{4}}{n^{2}}+\frac{L_{M}^{4}}{m^{2}}\right) .
$$

Now we show that conditions (3.9), (3.11) and (3.12) hold. Under the hypotheses of the theorem we have $M_{N}=n^{c}$ and $L_{M}=m^{c^{\prime}}$, where $c$ and $c^{\prime}$ are smaller than $1 / 2$. We choose $\sigma_{N}=n^{-d}$ and $\sigma_{M}^{\prime}=m^{-d^{\prime}}$ with $d=2-4 c$ and $d^{\prime}=2-4 c^{\prime}$. Then

$$
\operatorname{Var}\left[f_{N, M}\left(\lambda_{1}, \lambda_{2}\right)\right]=O\left(\frac{1}{n^{d}}+\frac{1}{m^{d^{\prime}}}\right) .
$$

We have taken $\delta(N)=n^{-\beta}$ and $\delta^{\prime}(M)=m^{-\beta^{\prime}}$ by choosing

$$
\beta=\frac{3 k \alpha-3 c k \alpha}{1+2 k \alpha} \quad \text { and } \quad \beta^{\prime}=\frac{3 k \alpha-3 c^{\prime} k \alpha}{1+2 k \alpha}
$$

and using the hypotheses of the theorem on $c$ and $c^{\prime}$, we show that the expressions of (3.9), (3.11) and (3.12) tend to zero with same rate. Thus we obtain

$$
\operatorname{Var}\left[f_{N, M}\left(\lambda_{1}, \lambda_{2}\right)\right]=O\left(\frac{1}{n^{2(1-2 c)}}+\frac{1}{m^{2\left(1-2 c^{\prime}\right)}}\right) .
$$

Theorem 3.4. Let $\lambda_{1}, \lambda_{2} \in \mathcal{A}$ be such that $\phi\left(\lambda_{1}, \lambda_{2}\right) \neq 0$. Then:

(i) $\mathbf{E}\left|f_{N, M}\left(\lambda_{1}, \lambda_{2}\right)-\left[\phi\left(\lambda_{1}, \lambda_{2}\right)\right]^{p / \alpha}\right|^{2}=o(1)$.

(ii) Under the conditions of Theorem 3.3(ii), for $k$ satisfying $\gamma+1<2 k \alpha$ we have

$$
\begin{aligned}
& \mathbf{E}\left|f_{N, M}\left(\lambda_{1}, \lambda_{2}\right)-\left[\phi\left(\lambda_{1}, \lambda_{2}\right)\right]^{p / \alpha}\right|^{2} \\
& = \begin{cases}O\left(\frac{1}{n^{2(1-2 c)}}+\frac{1}{m^{2\left(1-2 c^{\prime}\right)}}+\left[\frac{1}{M_{N}^{\gamma}}+\frac{1}{L_{M}^{\gamma}}\right]^{2}\right) & \text { if } \phi \text { satisfies }\left(h_{1}\right), \\
O\left(\frac{1}{n^{2(1-2 c)}}+\frac{1}{m^{2\left(1-2 c^{\prime}\right)}}+\left[\frac{1}{M_{N}}+\frac{1}{L_{M}}\right]^{2}\right) & \text { if } \phi \text { satisfies }\left(h_{2}\right) .\end{cases}
\end{aligned}
$$


Proof. It is easy to show that

$$
\begin{aligned}
\mathbf{E} \mid f_{N, M}\left(\lambda_{1}, \lambda_{2}\right) & -\left.\left[\phi\left(\lambda_{1}, \lambda_{2}\right)\right]^{p / \alpha}\right|^{2} \\
& \left.=\left(\mathbf{E}\left[f_{N, M}\left(\lambda_{1}, \lambda_{2}\right)\right]-\left[\phi\left(\lambda_{1}, \lambda_{2}\right)\right]^{p / \alpha}\right]\right)^{2}-\operatorname{Var}\left[f_{N, M}\left(\lambda_{1}, \lambda_{2}\right)\right] .
\end{aligned}
$$

Theorems 3.1 and 3.3 give the result.

TheOREM 3.5. Let $\lambda_{1}, \lambda_{2} \in \mathcal{A}$. Then $\left[f_{N, M}\left(\lambda_{1}, \lambda_{2}\right)\right]^{\alpha / p}$ converges in probability to $\phi\left(\lambda_{1}, \lambda_{2}\right)$.

Proof. As in the work of Masry and Cambanis [11], we use the inequality

$$
\left|y^{q}-x^{q}\right| \leq \frac{q}{2}|y-x|\left(y^{q-1}+x^{q-1}\right), \quad x, y \in \mathbb{R}^{+}, q>2,
$$

to obtain

$\left|\left[f_{N, M}\left(\lambda_{1}, \lambda_{2}\right)\right]^{\alpha / p}-\phi\left(\lambda_{1}, \lambda_{2}\right)\right|$

$\leq \frac{\alpha}{2 p}\left|f_{N, M}\left(\lambda_{1}, \lambda_{2}\right)-\left[\phi\left(\lambda_{1}, \lambda_{2}\right)\right]^{p / \alpha}\right|\left(\left[f_{N, M}\left(\lambda_{1}, \lambda_{2}\right)\right]^{\alpha / p-1}-\left[\phi\left(\lambda_{1}, \lambda_{2}\right)\right]^{(\alpha-p) / \alpha}\right)$.

Then we show easily that $\left[f_{N, M}\left(\lambda_{1}, \lambda_{2}\right)\right]^{\alpha / p}$ converges in probability to $\phi\left(\lambda_{1}, \lambda_{2}\right)$.

4. The double kernel method. It remains to estimate $\phi$ at points $\left(\lambda_{1}, \lambda_{2}\right)$ which belong to $]-\pi, \pi\left[^{2}\right.$ but do not belong to $\mathcal{A}$. To do that, we use the double kernel method, introduced by Priestley in [12], for processes of second order. We consider a nonnegative, even and continuous function $W$ vanishing for $|\lambda|>1$, with $\int_{-1}^{1} W(v) d v=1$ and $W(0) \neq 0$. We define the spectral windows:

$$
\begin{array}{ll}
W_{N}^{(1)}(\lambda)=M_{N}^{(1)} W\left(\lambda M_{N}^{(1)}\right), & W_{N}^{(2)}(\lambda)=M_{N}^{(2)} W\left(\lambda M_{N}^{(2)}\right), \\
W_{M}^{(1)}(\lambda)=L_{M}^{(1)} W\left(\lambda L_{M}^{(1)}\right), & W_{M}^{(2)}(\lambda)=L_{M}^{(2)} W\left(\lambda L_{M}^{(2)}\right),
\end{array}
$$

where $M_{N}^{(1)}, M_{N}^{(2)}, L_{M}^{(1)}$ and $L_{M}^{(2)}$ satisfy

$$
\begin{gathered}
\lim _{N \rightarrow \infty} M_{N}^{(i)}=\infty, \quad \lim _{M \rightarrow \infty} L_{M}^{(i)}=\infty, \\
\lim _{N \rightarrow \infty} M_{N}^{(i)} / N=0, \quad \lim _{M \rightarrow \infty} L_{M}^{(i)} / M=0, \quad i=1,2,
\end{gathered}
$$

and

$$
\lim _{N \rightarrow \infty} M_{N}^{(2)} / M_{N}^{(1)}=0, \quad \lim _{M \rightarrow \infty} L_{M}^{(2)} / L_{M}^{(1)}=0 .
$$

We suppose that $\left(M_{N}^{(1)}\right)^{2 k \alpha} / n^{2 k \alpha-1}$ and $\left(L_{M}^{(1)}\right)^{2 k \alpha} / m^{2 k \alpha-1}$ converge to 0 . For example, $M_{N}^{(1)}=n^{b}, M_{N}^{(2)}=n^{c}$ with $0<c<b<1-1 /(2 k \alpha)$. These spectral windows must be such that there exist nonnegative real numbers $c$ 
and $c^{\prime}$ different from 0 and 1 , satisfying the relations

$$
\begin{aligned}
W_{N}^{(2)}(\theta)-c W_{N}^{(1)}(\theta)=0, & -1 / M_{N}^{(1)}<\theta<1 / M_{N}^{(1)}, \\
W_{M}^{(2)}\left(\theta^{\prime}\right)-c^{\prime} W_{M}^{(1)}\left(\theta^{\prime}\right)=0, & -1 / L_{M}^{(1)}<\theta^{\prime}<1 / L_{M}^{(1)} .
\end{aligned}
$$

Consequently, $c=M_{N}^{(2)} / M_{N}^{(1)}$ and $c^{\prime}=L_{M}^{(2)} / L_{M}^{(1)}$. Let

$$
\begin{aligned}
f_{N, M}^{(1)}\left(\lambda_{1}, \lambda_{2}\right)= & \int_{-\pi}^{\pi} \int_{-\pi}^{\pi} W_{N}^{(1)}\left(\lambda_{1}-u_{1}\right) W_{M}^{(1)}\left(\lambda_{2}-u_{2}\right) \widehat{I}_{N, M}\left(u_{1}, u_{2}\right) d u_{1} d u_{2}, \\
f_{N, M}^{(2)}\left(\lambda_{1}, \lambda_{2}\right)= & \int_{-\pi}^{\pi} \int_{-\pi}^{\pi}\left(\frac{W_{N}^{(2)}\left(\lambda_{1}-u_{1}\right)-c W_{N}^{(1)}\left(\lambda_{1}-u_{1}\right)}{1-c}\right) \\
& \times\left(\frac{W_{M}^{(2)}\left(\lambda_{2}-u_{2}\right)-c^{\prime} W_{M}^{(1)}\left(\lambda_{2}-u_{2}\right)}{1-c^{\prime}}\right) \widehat{I}_{N, M}\left(u_{1}, u_{2}\right) d u_{1} d u_{2} .
\end{aligned}
$$

We consider the following estimator:

$$
f_{N, M}\left(\lambda_{1}, \lambda_{2}\right)= \begin{cases}f_{N, M}^{(1)}\left(\lambda_{1}, \lambda_{2}\right) & \text { when }\left(\lambda_{1}, \lambda_{2}\right) \in \mathcal{A}, \\ f_{N, M}^{(2)}\left(\lambda_{1}, \lambda_{2}\right) & \text { elsewhere. }\end{cases}
$$

Theorem 4.1. Let $\left.\lambda_{1}, \lambda_{2} \in\right]-\pi, \pi\left[\right.$. Then $f_{N, M}\left(\lambda_{1}, \lambda_{2}\right)$ is an asymptotically unbiased estimator of $\left[\phi\left(\lambda_{1}, \lambda_{2}\right)\right]^{p / \alpha}$.

Pr o of. We only study the case where $\left(\lambda_{1}, \lambda_{2}\right) \notin \mathcal{A}$, the other cases are considered in Theorem 3.1. We show easily that for $N$ and $M$ large enough,

$$
\begin{array}{r}
\int_{-\pi}^{\pi} \int_{-\pi}^{\pi}\left(\frac{W_{N}^{(2)}\left(\lambda_{1}-u_{1}\right)-c W_{N}^{(1)}\left(\lambda_{1}-u_{1}\right)}{1-c}\right) \\
\times\left(\frac{W_{M}^{(2)}\left(\lambda_{2}-u_{2}\right)-c^{\prime} W_{M}^{(1)}\left(\lambda_{2}-u_{2}\right)}{1-c^{\prime}}\right) d u_{1} d u_{2}=1 .
\end{array}
$$

Our choice of $W_{N}^{(1)}$ and $W_{N}^{(2)}$ implies that, for large $N$ and $M$,

$$
\begin{aligned}
\mathbf{E}\left[f_{N, M}^{(2)}\left(\lambda_{1}, \lambda_{2}\right)\right]-\left[\phi\left(\lambda_{1}, \lambda_{2}\right)\right]^{p / \alpha} & \\
= & \sum_{k, p=1}^{2} \frac{1}{(1-c)\left(1-c^{\prime}\right)} \int_{M_{N}^{(2)} / M_{N}^{(1)}}^{1} \int_{L_{M}^{(2)} / L_{M}^{(1)}}^{1} W\left(v_{1}\right) W\left(v_{2}\right) \\
\times & {\left[\left[\psi_{N, M}\left(\lambda_{1}+(-1)^{p} v_{1} / M_{N}^{(2)}, \lambda_{2}+(-1)^{k} v_{2} / L_{M}^{(2)}\right)\right]^{p / \alpha}\right.} \\
- & {\left.\left[\phi\left(\lambda_{1}, \lambda_{2}\right)\right]^{p / \alpha}\right] d v_{1} d v_{2} . }
\end{aligned}
$$


We are going to show that the following quantity converges to zero:

$$
\begin{aligned}
E^{\prime}= & \frac{1}{(1-c)\left(1-c^{\prime}\right)} \int_{M_{N}^{(2)} / M_{N}^{(1)}}^{1} \int_{L_{M}^{(2)} / L_{M}^{(1)}}^{1} W\left(v_{1}\right) W\left(v_{2}\right) \\
& \times\left[\left[\psi_{N, M}\left(\lambda_{1} \pm v_{1} / M_{N}^{(2)}, \lambda_{2} \pm v_{2} / L_{M}^{(2)}\right)\right]^{p / \alpha}-\left[\phi\left(\lambda_{1}, \lambda_{2}\right)\right]^{p / \alpha}\right] d v_{1} d v_{2} .
\end{aligned}
$$

Since $p / \alpha<1$, we obtain

$$
\begin{aligned}
\left|E^{\prime}\right| \leq & \frac{1}{(1-c)\left(1-c^{\prime}\right)} \int_{M_{N}^{(2)} / M_{N}^{(1)}}^{1} \int_{L_{M}^{(2)} / L_{M}^{(1)}}^{1} W\left(v_{1}\right) W\left(v_{2}\right) \\
& \times\left|\psi_{N, M}\left(\lambda_{1} \pm v_{1} / M_{N}^{(2)}, \lambda_{2} \pm v_{2} / L_{M}^{(2)}\right)-\phi\left(\lambda_{1}, \lambda_{2}\right)\right|^{p / \alpha} d v_{1} d v_{2} .
\end{aligned}
$$

Thus from (3.4) and the definition of $\psi_{N, M}$ we have

where

$$
\left|E^{\prime}\right| \leq \frac{1}{(1-c)\left(1-c^{\prime}\right)}\left[E_{1}^{\prime}+E_{2}^{\prime}+E_{3}^{\prime}\right]
$$

$$
\begin{aligned}
E_{1}^{\prime}= & \int_{M_{N}^{(2)} / M_{N}^{(1)}}^{1} \int_{L_{M}^{(2)} / L_{M}^{(1)}}^{1} W\left(v_{1}\right) W\left(v_{2}\right) \\
& \times\left|I_{N, M}\left(\lambda_{1} \pm v_{1} / M_{N}^{(2)}, \lambda_{2} \pm v_{2} / L_{M}^{(2)}\right)-\phi\left(\lambda_{1}, \lambda_{2}\right)\right|^{p / \alpha} d v_{1} d v_{2}, \\
E_{2}^{\prime}= & \int_{M_{N}^{(2)} / M_{N}^{(1)}}^{1} \int_{L_{M}^{(2)} / L_{M}^{(1)}}^{1} W\left(v_{1}\right) W\left(v_{2}\right) \\
& \times\left|J_{N, M}\left(\lambda_{1} \pm v_{1} / M_{N}^{(2)}, \lambda_{2} \pm v_{2} / L_{M}^{(2)}\right)\right|^{p / \alpha} d v_{1} d v_{2} \\
E_{3}^{\prime}= & \int^{1} \int_{N}^{(2)} / M_{N}^{(1)} L_{M}^{(2)} / L_{M}^{(1)} \\
& \times\left|K_{N, M}\left(\lambda_{1} \pm v_{1} / M_{N}^{(2)}, \lambda_{2} \pm v_{2} / L_{M}^{(2)}\right)\right|^{p / \alpha} d v_{1} d v_{2} .
\end{aligned}
$$

Since $M_{N}^{(1)} / M_{N}^{(2)}>0$ and $L_{M}^{(1)} / L_{M}^{(2)}>0$, from (3.1) we see that $I_{N, M}\left(\lambda_{1} \pm\right.$ $\left.v_{1} / M_{N}^{(2)}, \lambda_{2} \pm v_{2} / L_{M}^{(2)}\right)$ converges to $\phi\left(\lambda_{1}, \lambda_{2}\right)$ uniformly in $v_{1}, v_{2} \in[-1,1]$. Hence $E_{1}^{\prime}$ converges to zero.

For $E_{2}^{\prime}$ we use Hölder's inequality and (3.4) to obtain

$$
\begin{aligned}
E_{2}^{\prime} \leq & {[\sup (W)]^{2} \sum_{j=1}^{q}\left(a_{j}^{\prime} \int_{M_{N}^{(2)} / M_{N}^{(1)}}^{1}\left|H_{N}\left(\lambda_{1} \pm v_{1} / M_{N}^{(2)}-w_{1 j}\right)\right|^{\alpha} d v_{1}\right)^{p / \alpha} } \\
& \times\left(\int_{L_{M}^{(2)} / L_{M}^{(1)}}^{1}\left|H_{M}\left(\lambda_{2} \pm v_{2} / L_{M}^{(2)}-w_{2 j}\right)\right|^{\alpha} d v_{2}\right)^{p / \alpha} \cdot
\end{aligned}
$$


We distinguish two cases:

Case 1: $\lambda_{1}=w_{1 j}$. Let

Then

$$
E_{2,1}^{\prime}=\int_{M_{N}^{(2)} / M_{N}^{(1)}}^{1}\left|H_{N}\left(\lambda_{1} \pm v_{1} / M_{N}^{(2)}-w_{1 j}\right)\right|^{\alpha} d v_{1} .
$$

$$
E_{2,1}^{\prime} \leq \frac{1}{B_{\alpha, N}^{\prime}} \int_{M_{N}^{(2)} / M_{N}^{(1)}}^{1} \frac{1}{\left.\sin \left(v_{1} /\left(2 M_{N}^{(2)}\right)\right)\right|^{2 k \alpha}} d v_{1}
$$

Therefore for large $N$ we have $0<1 / M_{N}^{(1)} \leq v_{1} / M_{N}^{(2)} \leq 1 / M_{N}^{(2)}<\pi$ and from (3.10) we get

$$
E_{2,1}^{\prime} \leq \frac{1}{B_{\alpha, N}^{\prime}}\left(1-\frac{M_{N}^{(2)}}{M_{N}^{(1)}}\right)(2 \pi)^{2 k \alpha}\left(M_{N}^{(1)}\right)^{2 k \alpha} .
$$

From Lemma 2.1, we obtain

$$
E_{2,1}^{\prime}=O\left(\frac{\left(M_{N}^{(1)}\right)^{2 k \alpha}}{n^{2 k \alpha-1}}\right)
$$

Similarly we have $E_{2,1}^{\prime}=O\left(1 / n^{2 k \alpha-1}\right)$. Now,

$$
\begin{aligned}
& \int_{L_{M}^{(2)} / L_{M}^{(1)}}^{1} \mid H_{M}\left(\lambda_{2} \pm v_{2} / L_{M}^{(2)}\right.\left.-w_{2 j}\right)\left.\right|^{\alpha} d v_{2} \\
& \leq \mathrm{const} \begin{cases}\left(L_{M}^{(1)}\right)^{2 k \alpha} / m^{2 k \alpha-1} & \text { if } \lambda_{2}=w_{2 j}, \\
1 / m^{2 k \alpha-1} & \text { if } \lambda_{2} \neq w_{2 j} .\end{cases}
\end{aligned}
$$

Thus $E_{2}^{\prime}$ tends to zero. For $E_{3}^{\prime}$, we use again Hölder's inequality and (3.4) to get

$$
\begin{aligned}
E_{3}^{\prime} \leq & {[\sup (W)]^{2} \sum_{i=1}^{q^{\prime}}\left(a_{i}^{\prime}\right)^{p / \alpha}\left(\int_{M_{N}^{(2)} / M_{N}^{(1)}}^{1} \int_{M}^{(2)} / L_{M}^{(1)}\left[\int_{-\pi}^{\pi} \mid H_{N}\left(\lambda_{1} \pm v_{1} / M_{N}^{(2)}-t_{1}\right)\right.\right.} \\
& \left.\left.\times\left. H_{M}\left(\lambda_{2} \pm v_{2} / L_{M}^{(2)}-a_{i} t_{1}-b_{i}\right)\right|^{\alpha} d t_{1}\right] d v_{1} d v_{2}\right)^{p / \alpha} .
\end{aligned}
$$

Case 2: $\lambda_{2}-a_{i} \lambda_{1}-b_{i}=2 \beta \pi$ with $\beta \in \mathbb{Z}$. Since $H_{M}$ is $2 \pi$-periodic, it follows that

$$
\begin{aligned}
\int_{-\pi}^{\pi} \mid & \left.H_{N}\left(\lambda_{1} \pm v_{1} / M_{N}^{(2)}-t_{1}\right) H_{M}\left(\lambda_{2} \pm v_{2} / L_{M}^{(2)}-a_{i} t_{1}-b_{i}\right)\right|^{\alpha} d t_{1} \\
= & \int_{-\pi}^{\pi}\left|H_{N}\left(\lambda_{1} \pm v_{1} / M_{N}^{(2)}-t_{1}\right) H_{M}\left(a_{i} \lambda_{1} \pm v_{2} / L_{M}^{(2)}-a_{i} t_{1}\right)\right|^{\alpha} d t_{1} .
\end{aligned}
$$


Now $\left|H_{N}\right|^{\alpha}$ is a kernel and the function $t_{1} \rightarrow\left|H_{M}\left(a_{i} \lambda_{1} \pm v_{2} / L_{M}^{(2)}-a_{i} t_{1}\right)\right|^{\alpha}$ is continuous on $[-\pi, \pi]$. Therefore, fixing $M$ and letting $N \rightarrow \infty$, we obtain

$$
\begin{aligned}
\lim _{N \rightarrow \infty} \int_{-\pi}^{\pi}\left|H_{N}\left(\lambda_{1} \pm v_{1} / M_{N}^{(2)}-t_{1}\right) H_{M}\left(a_{i} \lambda_{1} \pm v_{2} / L_{M}^{(2)}-a_{i} t_{1}\right)\right|^{\alpha} d t_{1} \\
=\left|H_{M}\left(v_{2} / L_{M}^{(2)}\right)\right|^{\alpha},
\end{aligned}
$$

uniformly in $v_{1} \in[-1,1]$.

By the same arguments as for (4.3) we get

$$
\int_{L_{M}^{(2)} / L_{M}^{(1)}}^{1}\left|H_{M}\left(v_{2} / L_{M}^{(2)}\right)\right|^{\alpha} d v_{2} \leq \frac{1-L_{M}^{(2)} / L_{M}^{(1)}}{B_{\alpha, M}^{\prime}}\left(2 \pi L_{M}^{(1)}\right)^{2 k \alpha}=O\left(\frac{\left(L_{M}^{(1)}\right)^{2 k \alpha}}{m^{2 k \alpha-1}}\right) .
$$

Thus $E_{3}^{\prime}$ tends to zero.

Theorem 4.2. Let $\left.\lambda_{1}, \lambda_{2} \in\right]-\pi, \pi\left[\right.$ with $\phi\left(\lambda_{1}, \lambda_{2}\right)>0$. Then

$$
\operatorname{Var}\left[f_{N, M}\left(\lambda_{1}, \lambda_{2}\right)\right]=o(1) .
$$

Pr o o f. We study only the case where $\left(\lambda_{1}, \lambda_{2}\right) \notin \mathcal{A}$. In the same manner as for (4.1), we obtain

$$
\begin{aligned}
\operatorname{Var}[ & \left.f_{N, M}\left(\lambda_{1}, \lambda_{2}\right)\right] \\
= & \frac{1}{(1-c)\left(1-c^{\prime}\right)} \mathbf{E}\left[\sum_{k, k^{\prime}=1}^{2} \int_{M_{N}^{(2)} / M_{N}^{(1)}}^{1} \int_{L_{M}^{(2)} / L_{M}^{(1)}}^{1} W\left(v_{1}\right) W\left(v_{2}\right)\right. \\
& \times\left[\widehat{I}_{N, M}\left(\lambda_{1}+(-1)^{k} v_{1} / M_{N}^{(2)}, \lambda_{2}+(-1)^{k^{\prime}} v_{2} / L_{M}^{(2)}\right)\right. \\
& \left.\left.-\mathbf{E} \widehat{I}_{N, M}\left(\lambda_{1}+(-1)^{k} v_{1} / M_{N}^{(2)}, \lambda_{2}+(-1)^{k^{\prime}} v_{2} / L_{M}^{(2)}\right)\right] d v_{1} d v_{2}\right]^{2} .
\end{aligned}
$$

For simplicity we write

$$
\operatorname{Var}\left[f_{N, M}\left(\lambda_{1}, \lambda_{2}\right)\right]=\frac{1}{(1-c)\left(1-c^{\prime}\right)} \sum_{k, k^{\prime}, p, p^{\prime}=1}^{2} A\left(k, k^{\prime}, p, p^{\prime}\right),
$$

where

$$
\begin{aligned}
& A\left(k, k^{\prime}, p, p^{\prime}\right)= \int_{M_{N}^{(2)} / M_{N}^{(1)}}^{1} \int_{L_{M}^{(2)} / L_{M}^{(1)}}^{1} \int_{M_{N}^{(2)} / M_{N}^{(1)}}^{1} \int_{L_{M}^{(2)} / L_{M}^{(1)}}^{1} W\left(v_{1}\right) W\left(v_{2}\right) W\left(v_{1}^{\prime}\right) W\left(v_{2}^{\prime}\right) \\
& \times \operatorname{Cov}\left[\widehat{I}_{N, M}\left(\lambda_{1}+(-1)^{k} v_{1} / M_{N}^{(2)}, \lambda_{2}+(-1)^{k^{\prime}} v_{2} / L_{M}^{(2)}\right),\right. \\
&\left.\widehat{I}_{N, M}\left(\lambda_{1}+(-1)^{p} v_{1}^{\prime} / M_{N}^{(2)}, \lambda_{2}+(-1)^{p^{\prime}} v_{2}^{\prime} / L_{M}^{(2)}\right)\right] d v_{1} d v_{2} d v_{1}^{\prime} d v_{2}^{\prime} .
\end{aligned}
$$


We set

$$
\begin{aligned}
& \mathcal{L}_{1, N}=\left\{\left(v_{1}, v_{1}^{\prime}\right) \in\left[M_{N}^{(2)} / M_{N}^{(1)}, 1\right]^{2}:\left|(-1)^{k} v_{1}-(-1)^{p} v_{1}^{\prime}\right|>\sigma_{N}\right\}, \\
& \mathcal{L}_{2, N}=\left\{\left(v_{2}, v_{2}^{\prime}\right) \in\left[L_{M}^{(2)} / L_{M}^{(1)}, 1\right]^{2}:\left|(-1)^{k^{\prime}} v_{2}-(-1)^{p^{\prime}} v_{2}^{\prime}\right|>\sigma_{M}^{\prime}\right\}, \\
& \mathcal{L}_{3, N}=\left\{\left(v_{1}, v_{1}^{\prime}, v_{2}, v_{2}^{\prime}\right) \in\left[M_{N}^{(2)} / M_{N}^{(1)}, 1\right]^{2} \times\left[L_{M}^{(2)} / L_{M}^{(1)}, 1\right]^{2}:\right. \\
&\left.\left|(-1)^{k} v_{1}-(-1)^{p} v_{1}^{\prime}\right| \leq \sigma_{N} \text { or }\left|(-1)^{k^{\prime}} v_{2}-(-1)^{p^{\prime}} v_{2}^{\prime}\right| \leq \sigma_{M}^{\prime}\right\},
\end{aligned}
$$

where $\sigma_{N}$ and $\sigma_{M}^{\prime}$ are two nonnegative real sequences converging to 0 . We have

$$
A\left(k, k^{\prime}, p, p^{\prime}\right)=\iint_{\mathcal{L}_{3, N}} \iint_{\mathcal{L}_{1, N}} \int_{\mathcal{L}_{2, N}} \int_{\mathcal{L}_{2}}=: J_{1}^{\prime}+J_{2}^{\prime}
$$

Using (3.4), we obtain

$$
\begin{aligned}
\operatorname{Var}\left[\widehat{I}_{N, M}\right. & \left.\left(\lambda_{1}+(-1)^{k} v_{1} / M_{N}^{(2)}, \lambda_{2}+(-1)^{k^{\prime}} v_{2} / L_{M}^{(2)}\right)\right] \\
= & V_{\alpha, p}\left[I_{N, M}\left(\lambda_{1}+(-1)^{k} v_{1} / M_{N}^{(2)}, \lambda_{2}+(-1)^{k^{\prime}} v_{2} / L_{M}^{(2)}\right)\right]^{2 p / \alpha} \\
& +V_{\alpha, p}\left[J_{N, M}\left(\lambda_{1}+(-1)^{k} v_{1} / M_{N}^{(2)}, \lambda_{2}+(-1)^{k^{\prime}} v_{2} / L_{M}^{(2)}\right)\right]^{2 p / \alpha} \\
& +V_{\alpha, p}\left[K_{N, M}\left(\lambda_{1}+(-1)^{k} v_{1} / M_{N}^{(2)}, \lambda_{2}+(-1)^{k^{\prime}} v_{2} / L_{M}^{(2)}\right)\right]^{2 p / \alpha}
\end{aligned}
$$

From (3.1), $\left[I_{N, M}\left(\lambda_{1}+(-1)^{k} v_{1} / M_{N}^{(2)}, \lambda_{2}+(-1)^{k^{\prime}} v_{2} / L_{M}^{(2)}\right)\right]^{2 p / \alpha}$ converges to $\left[\phi\left(\lambda_{1}, \lambda_{2}\right)\right]^{2 p / \alpha}$ uniformly in $v_{1}, v_{2} \in[-1,1]$. For $N$ and $M$ large enough we obtain

$$
\begin{aligned}
& \int_{\mathcal{L}_{3, N}} W\left(v_{1}\right) W\left(v_{2}\right) W\left(v_{1}^{\prime}\right) W\left(v_{2}^{\prime}\right) \\
& \quad \times\left[I_{N, M}\left(\lambda_{1}+(-1)^{k} v_{1} / M_{N}^{(2)}, \lambda_{2}+(-1)^{k^{\prime}} v_{2} / L_{M}^{(2)}\right)\right]^{2 p / \alpha} d v_{1} d v_{1}^{\prime} d v_{2} d v_{2}^{\prime} \\
& \quad \leq \operatorname{const}\left[\sigma_{N}+\sigma_{M}^{\prime}\right] .
\end{aligned}
$$

Using inequality (3.4),

$$
\begin{aligned}
{\left[J _ { N , M } \left(\lambda_{1}+(-1)^{k} v_{1} / M_{N}^{(2)}\right.\right.} & \left.\left., \lambda_{2}+(-1)^{k^{\prime}} v_{2} / L_{M}^{(2)}\right)\right]^{2 p / \alpha} \\
\leq & \sum_{j=1}^{q}\left[\left|H_{N}\left(\lambda_{1}+(-1)^{k} v_{1} / M_{N}^{(2)}-w_{1 j}\right)\right|^{\alpha}\right. \\
& \left.\times\left|H_{M}\left(\lambda_{2}+(-1)^{k^{\prime}} v_{2} / L_{M}^{(2)}-w_{2 j}\right)\right|^{\alpha}\right]^{2 p / \alpha}
\end{aligned}
$$

We distinguish three cases:

Case 1: there exists $j \in\{1, \ldots, q\}$ such that $\lambda_{1}=w_{1 j}$. Then

$$
\left|H_{N}\left(\lambda_{1}+(-1)^{k} v_{1} / M_{N}^{(2)}-w_{1 j}\right)\right|^{\alpha} \leq \frac{1}{B_{\alpha, N}^{\prime}} \cdot \frac{1}{\left|\sin \left[\frac{1}{2}\left((-1)^{k} v_{1} / M_{N}^{(2)}\right)\right]\right|^{2 k \alpha}} \text {. }
$$


By using inequality (3.10), for large $N$ we get

$$
\frac{1}{\left|\sin \left[\frac{1}{2}\left((-1)^{k} v_{1} / M_{N}^{(2)}\right)\right]\right|^{2 k \alpha}} \leq \frac{1}{\left|\sin \left[1 /\left(2 M_{N}^{(1)}\right)\right]\right|^{2 k \alpha}} \leq \frac{\pi^{2 k \alpha}}{\left|1 /\left(2 M_{N}^{(1)}\right)\right|^{2 k \alpha}} .
$$

From Lemma 2.1, we obtain

$$
\left|H_{N}\left(\lambda_{1}+(-1)^{k} v_{1} / M_{N}^{(2)}-w_{1 j}\right)\right|^{\alpha}=O\left(\frac{\left(M_{N}^{(1)}\right)^{2 k \alpha}}{n^{2 k \alpha-1}}\right) .
$$

Case 2: $\lambda_{2}-a_{i} \lambda_{1}-b_{i} \notin 2 \pi \mathbb{Z}$ for every $i \in\left\{1, \ldots, q^{\prime}\right\}$. From (3.3), we get

$$
\begin{aligned}
\int_{-\pi}^{\pi} \mid H_{N}\left(\lambda_{1}+(-1)^{k}\right. & \left.v_{1} / M_{N}^{(2)}-v\right)\left.\right|^{\alpha} \\
& \times\left|H_{M}\left(\lambda_{2}+(-1)^{k^{\prime}} v_{2} / L_{M}^{(2)}-a_{i} v-b_{i}\right)\right|^{\alpha} \phi_{i}(v) d v \\
= & O\left(\frac{1}{n^{2 k \alpha-1}}+\frac{1}{n^{2 k \alpha-1} m^{2 k \alpha-1}}\right) .
\end{aligned}
$$

Case 3 : there exists $i \in\left\{1, \ldots, q^{\prime}\right\}$ such that $\lambda_{2}-a_{i} \lambda_{1}-b_{i}=2 \beta \pi$ with $\beta \in \mathbb{Z}$. Because $H_{M}$ is $2 \pi$-periodic and $\phi_{i}$ is bounded on $[-\pi, \pi]$, we obtain

$$
\begin{aligned}
& \int_{-\pi}^{\pi} \mid H_{N}\left(\lambda_{1}+(-1)^{k}\right.\left.v_{1} / M_{N}^{(2)}-v\right)\left.\right|^{\alpha} \\
& \times\left|H_{M}\left(\lambda_{2}+(-1)^{k^{\prime}} v_{2} / L_{M}^{(2)}-a_{i} v-b_{i}\right)\right|^{\alpha} \phi_{i}(v) d v \\
& \leq \sup \left(\phi_{i}\right) \int_{-\pi}^{\pi}\left|H_{N}\left(\lambda_{1}+(-1)^{k} v_{1} / M_{N}^{(2)}-v\right)\right|^{\alpha} \\
& \times\left|H_{M}\left(a_{i} \lambda_{1}+(-1)^{k^{\prime}} v_{2} / L_{M}^{(2)}-a_{i} v\right)\right|^{\alpha} d v
\end{aligned}
$$

Now $\left|H_{N}\right|^{\alpha}$ is a kernel and the function $t \rightarrow \mid H_{M}\left(a_{i} \lambda_{1}+(-1)^{k^{\prime}} v_{2} / L_{M}^{(2)}-\right.$ $\left.a_{i} t\right)\left.\right|^{\alpha}$ is continuous on $[-\pi, \pi]$. Hence, as $N \rightarrow \infty$, the last integral tends to $\left|H_{M}\left((-1)^{k^{\prime}} v_{2} / L_{M}^{(2)}\right)\right|^{\alpha}$. Thus for $M$ large enough we obtain

$$
\left|H_{M}\left((-1)^{k^{\prime}} v_{2} / L_{M}^{(2)}\right)\right|^{\alpha} \leq \frac{1}{B_{\alpha, M}^{\prime}}(2 \pi)^{2 k \alpha}\left(L_{M}^{(1)}\right)^{2 k \alpha} .
$$

Therefore, $\left[K_{N, M}\left(\lambda_{1}+(-1)^{k} v_{1} / M_{N}^{(2)}, \lambda_{2}+(-1)^{k^{\prime}} v_{2} / L_{M}^{(2)}\right)\right]^{2 p / \alpha}$ converges to zero. Thus $\operatorname{Var}\left[\hat{I}_{N, M}\left(\lambda_{1}+(-1)^{k} v_{1} / M_{N}^{(2)}, \lambda_{2}+(-1)^{k^{\prime}} v_{2} / L_{M}^{(2)}\right)\right]$ tends to zero uniformly in $v_{1}, v_{2} \in[-1,1]$. Consequently, $J_{1}^{\prime}$ tends to zero.

On the other hand, for $J_{2}^{\prime}$ we define

$$
\begin{array}{ll}
\lambda_{1,1}=\lambda_{1}+(-1)^{k} v_{1} / M_{N}^{(2)}, & \lambda_{1,2}=\lambda_{1}+(-1)^{p} v_{1}^{\prime} / M_{N}^{(2)}, \\
\lambda_{2,1}=\lambda_{2}+(-1)^{k^{\prime}} v_{2} / L_{M}^{(2)}, & \lambda_{2,2}=\lambda_{2}+(-1)^{p^{\prime}} v_{2}^{\prime} / L_{M}^{(2)} .
\end{array}
$$


As in the previous section we obtain

$$
\begin{aligned}
J_{2}^{\prime} \leq & \int_{\mathcal{L}_{1, N}} \int_{\mathcal{L}_{2, N}} W\left(v_{1}\right) W\left(v_{2}\right) W\left(v_{1}^{\prime}\right) W\left(v_{2}^{\prime}\right) Q_{N, M}\left(\lambda_{1,1}, \lambda_{1,2}, \lambda_{2,1}, \lambda_{2,2}\right) \\
& \times\left(\int_{-\infty}^{\infty} \int_{-\infty}^{\infty} \frac{e^{\left|K-K^{\prime}\right|-K^{\prime}}}{\left|u_{1} u_{2}\right|^{1+p-\alpha / 2}} d u_{1} d u_{2}\right) d v_{1} d v_{1}^{\prime} d v_{2} d v_{2}^{\prime}
\end{aligned}
$$

where $K, K^{\prime}$ and $Q_{N, M}\left(\lambda_{1,1}, \lambda_{1,2}, \lambda_{2,1}, \lambda_{2,2}\right)$ are defined in Section 3. Analogously to the previous calculations, we show that

$$
\lim _{\substack{N \rightarrow \infty \\ M \rightarrow \infty}} Q_{N, M}\left(\lambda_{1,1}, \lambda_{1,2}, \lambda_{2,1}, \lambda_{2,2}\right)=0
$$

and

$$
\begin{aligned}
\lim _{\substack{N \rightarrow \infty \\
M \rightarrow \infty}} \int_{-\infty}^{\infty} \int_{-\infty}^{\infty} \frac{e^{\left|K-K^{\prime}\right|-K^{\prime}}}{\left|u_{1} u_{2}\right|^{1+p-\alpha / 2}} d u_{1} d u_{2} \\
\leq \frac{1}{\left[\phi\left(\lambda_{1}, \lambda_{2}\right)\right]^{1-2 p / \alpha}}\left(\int_{-\infty}^{\infty} \frac{e^{-C_{\alpha}|v|^{\alpha}}}{|v|^{1+p-\alpha / 2}} d v\right)^{2} .
\end{aligned}
$$

Thus $J_{2}^{\prime}$ tends to zero, and consequently $\operatorname{Var}\left[f_{N, M}\left(\lambda_{1}, \lambda_{2}\right)\right]$ converges to zero.

The following two theorems are proved with analogous methods.

TheOrem 4.3. Let $\left.\lambda_{1}, \lambda_{2} \in\right]-\pi, \pi\left[\right.$ and $\phi\left(\lambda_{1}, \lambda_{2}\right)>0$. Then

$$
\lim _{\substack{N \rightarrow \infty \\ M \rightarrow \infty}} \mathbf{E}\left|f_{N, M}\left(\lambda_{1}, \lambda_{2}\right)-\left[\phi\left(\lambda_{1}, \lambda_{2}\right)\right]^{p / \alpha}\right|^{2}=0
$$

TheOREM 4.4. Let $\left.\lambda_{1}, \lambda_{2} \in\right]-\pi, \pi\left[\right.$ and suppose that $\phi\left(\lambda_{1}, \lambda_{2}\right)>0$. Then $\left[f_{N, M}\left(\lambda_{1}, \lambda_{2}\right)\right]^{\alpha / p}$ converges in probability to $\phi\left(\lambda_{1}, \lambda_{2}\right)$.

\section{References}

[1] B. S. Rajput and J. Rosinski, Spectral representations of infinitely divisible processes, Probab. Theory Related Fields 82 (1989), 451-487.

[2] M. Bertrand-Retali, Processus symétriques $\alpha$-stables, Séminaire de Probabilité et Statistique à l'Université de Constantine, Algérie, 1987.

[3] S. Cambanis, Complex symmetric stable variables and processes, in: P. K. Sen (ed.), Contributions to Statistics: Essays in Honour of Norman L. Johnson, NorthHolland, New York, 1982, 63-79.

[4] S. Cambanis and G. Miller, Some path properties of pth order and symmetric stable processes, Ann. Probab. 8 (1980), 1148-1156.

[5] - - - Linear problems in pth order and stable processes, SIAM J. Appl. Math. 41 (1981), 43-69. 
[6] N. Demesh, Application of the polynomial kernels to the estimation of the spectra of discrete stable stationary processes, Akad. Nauk Ukrain. SSR, Inst. Mat. Preprint 64 (1988), 12-36 (in Russian).

[7] V. K. Dzyadyk, Introduction à la théorie de l'approximation uniforme par fonctions polynomiales, Nauka, 1977 (in Russian).

[8] C. D. Hardin, On the spectral representation theorem for symmetric stable processes, J. Multivariate Anal. 12 (1982), 385-401.

[9] L. Heinrich, On the convergence of U-statistics with stable limit distribution, J. Multivariate Anal. 44 (1993), 266-278.

[10] Y. Hosoya, Discrete-time stable processes and their certain properties, Ann. Probab. 6 (1978), 94-105.

[11] E. Masry and S. Cambanis, Spectral density estimation for stationary stable processes, Stochastic Process. Appl. 18 (1984), 1-31.

[12] M. B. Priestley, Spectral Analysis and Time Series, Probab. Math. Statist., Academic Press, 1981.

[13] R. Sabre, Estimation non paramétrique dans les processus symétriques stables, Thèse de doctorat en Mathématiques, Université de Rouen, 1993.

[14] M. Schilder, Some structure theorems for the symmetric stable laws, Ann. Math. Statist. 42 (1970), 412-421.

[15] R. Song, Probabilistic approach to the Dirichlet problem of perturbed stable processes, Probab. Theory Related Fields 95 (1993), 371-389.

RACHID SABRE

ETABLISSEMENT NATIONAL D'ENSEIGNEMENT SUPÉRIEUR

AGRONOMIQUE DE DIJON

26, BOULEVARD DU DR. PETITJEAN

B.P. 1607

F-21036 DIJON, FRANCE 